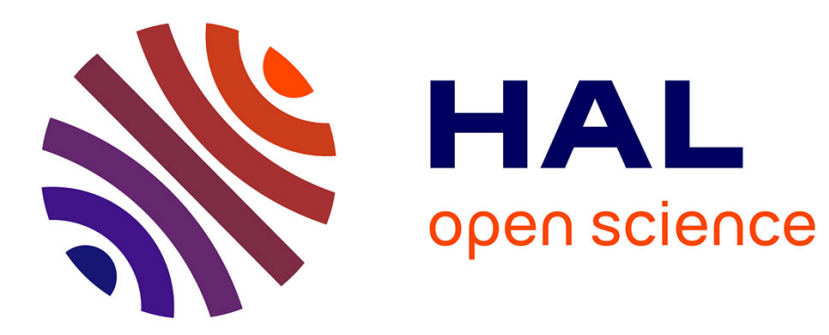

\title{
Estimation of local failure in tensegrity using Interacting Particle-Ensemble Kalman Filter
}

\author{
Neha Aswal, Subhamoy Sen, Laurent Mevel
}

\section{To cite this version:}

Neha Aswal, Subhamoy Sen, Laurent Mevel. Estimation of local failure in tensegrity using Interacting Particle-Ensemble Kalman Filter. Mechanical Systems and Signal Processing, 2021, 160, pp.107824. 10.1016/j.ymssp.2021.107824 . hal-03468255

\section{HAL Id: hal-03468255 \\ https://hal.inria.fr/hal-03468255}

Submitted on 7 Dec 2021

HAL is a multi-disciplinary open access archive for the deposit and dissemination of scientific research documents, whether they are published or not. The documents may come from teaching and research institutions in France or abroad, or from public or private research centers.
L'archive ouverte pluridisciplinaire HAL, est destinée au dépôt et à la diffusion de documents scientifiques de niveau recherche, publiés ou non, émanant des établissements d'enseignement et de recherche français ou étrangers, des laboratoires publics ou privés. 


\section{Estimation of local failure in tensegrity using Interacting Particle-Ensemble

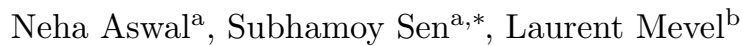

${ }^{a}$ Indian Institute of Technology Mandi, Mandi, HP, India

${ }^{b}$ Univ. Gustave Eiffel, Inria, Cosys-SII, I4S, Campus de Beaulieu, France

\section{Abstract}

7 Tensegrities form a special case of truss, wherein compression members (struts/bars) float within a network 8 of tension members (cables). Tensegrities are characterized by the presence of at least one infinitesimal 9 mechanism stabilized with member pre-stress to ensure equilibrium. Over prolonged usage, the cables may lose their pre-stress while the bars may buckle, get damaged, or corrode, affecting the structural stiffness 1 leading to change in the measured dynamic properties. Upon loading, a tensegrity structure may change

\footnotetext{
*Corresponding author; E-mail address: subhamoy@iitmandi.ac.in
} 
finds its origin in the works of Ioganson (1920) and Snelson (1948) [54, where Ioganson's structure lacks one of the essential criteria for tensegrity, i.e., equilibrium without any external force [57. While tensegrity was later formally introduced by Snelson as an architectural piece, its potential as a structure was promoted by Buckminster Fuller. Ever since its introduction, tensegrities have found applications in various fields: aerospace [68, bio-mechanics [34, robotics [44, etc. Although tensegrities demonstrate excellent utility for being deployable as well as aesthetically appealing [53, 64, the typical perspective of being considered as a light-weight structure has been debated in $[26$. Nevertheless, the unique deployable attribute of tensegrities has found much acceptance in the field of controllable structures [62, 63, 65, aerospace application [21, 41, 61] and especially in robotics [6, 20, 35] wherein tension in the strings/cables are actively controlled by actuators to control the movement of the tensegrity robots. Accordingly, various methodologies have been developed to design [5, 67] and construct statically stable complex tensegrity structures [60, 72, that are easy to erect or deploy. The success with non-load bearing structures has quickly been adapted by the structural engineers as well and the tensegrity concept has been implemented for civil infrastructures in the form of roofs and bridges. The motivation comes from the fact that tensegrities can provide large column-free spaces allowing sufficient overhead clearance (advantageous for bridges to allow water vessels underneath) and unobstructed view (beneficial for the stadium roofs) 2, 23, 25: Olympic Gymnastics Arena roof (Seoul, South Korea), Kurilpa bridge (Brisbane, Australia), etc. are some of the examples among many others.

Tensegrities are characterized by the presence of at least one infinitesimal mechanism [39] stiffened by the pre-stress present in the members due to their configuration. Of course, in the absence of these member pre-stresses, there would be no structure, thereby delineating tensegrities from other pre-stressed structures. The stability of tensegrity is therefore pre-stress dependant and conditioned on a particular configuration, known as self-stress configuration [69. To accommodate a certain external load, tensegrity incur changes in its initial stable configuration. Tensegrities can thus have multiple self-stressed stable configurations under different external loading conditions [49, 69]. Modification in the shape, due to pre-stress levels as well as external forces, eventually, changes the stiffness properties, thereby altering the frequencies even in its undamaged condition. Moreover, tensegrities can have same shape with different stiffness and frequencies, because of different pre-stress levels 3, 4. Tensegrities thus may exhibit different stiffness, dynamical properties, and spatial configurations even in its healthy state, which otherwise is anticipated only under damaged conditions for traditional structures. It should therefore be noted that stiffness alteration due to modification in member stress induced by force variability does not imply damage in a tensegrity.

As tensegrity does not belong to the category of traditional structures that are typically constructed with high levels of redundancy [25, the approach for monitoring its health is also not typical. Since tensegrities are substantially optimized [19, 56 from a design and construction point of view, a catastrophic failure may therefore occur if its health is not monitored rigorously. Moreover, their shape morphing attribute may lead to a false impression of damage when being dealt with traditional health monitoring techniques. Vibrational 
properties of tensegrities are contrastingly less explored [21, 38, 41, 42, than their static performance. Accordingly, studies related to vibration-based structural health monitoring (SHM) for tensegrities are also insufficient [4, 66]. Assessment of health from global parameters like modal information is not an option for tensegrity health monitoring since modal information keeps on changing even in its normal operational condition, discussed later in this article. Hence, to identify/monitor possible damages in tensegrity, it is important to have an SHM approach specific for tensegrity that takes into account its nature [49]. Yet literature available in this field is of an insignificant volume.

Three methods for tensegrity damage detection have been compared in [66], namely, frequency analysis, error-domain model falsification (EDMF) using node position measurement, and moving-window principal component analysis (MWPCA) using strain measurements. It has been observed that, for tensegrities, natural frequencies and mode shapes can not be considered as features sensitive only to damage (further demonstrated later in this article). The slacking scenario in the cables substantially impacts the first natural frequency, which however differs from one scenario to another. Hence to detect this reduction in tension, individual monitoring of damage induced frequency alterations has been suggested in [4]. Although it has been perceived that for tensegrities with forces unknown, modal domain SHM is no longer an option. Results obtained from EDMF [66] were observed to be sensitive to ambient uncertainty. Also, EDMF tends to become costly when tracking positions at sub-millimeter resolution. MWPCA 66, has an advantage over the other mentioned methods since it uses inexpensive strain gauges. It has been observed to be efficient with low to moderate noise levels but has been reported to perform poorly for high levels of noise contamination. Satisfactory performance for damage assessment using dynamic strain measurements has also been observed by [11. Electro-mechanical impedance (EMI) measures are also considered as measurements for this study, which has been analysed for high frequency signatures (in $\mathrm{kHz}$ ) as damage sensitive feature. The study further compares the performances of EMI and dynamic strain as measurements and concludes that the dynamic strain measurement-based approach is more cost-effective than the former.

Evidently, most of the works on tensegrity SHM have been cast in the deterministic domain. Nevertheless, any typical model-based SHM approach for a real tensegrity needs to deal with uncertainties due to modeling error, ambient forcing, and measurement noise. Yet these sources of uncertainties are mostly left unaccounted for with deterministic SHM approaches. Force is an important aspect of tensegrity stiffness and should thus be known for the deterministic tensegrity SHM approach to alienate a force-induced change in structural response from a damaged induced anomaly. For tensegrities, subjected to ambient force, the problem gets aggravated since an explicit knowledge of ambient forces is rarely available. Real-life tensegrities, therefore, need a special SHM approach capable of dealing with the forcing uncertainties efficiently.

In this context, Bayesian filters have proved their merit in SHM research dealing with the mentioned uncertainties. With Bayesian filter-based SHM approaches, the uncertainties due to force and modeling inaccuracies are dealt with a probabilistic process model while a measurement model deals with the sensor 
noise uncertainties separately. Within the process model, the dynamics of the structure is defined in statespace with a set of internal unobserved variables, called states. The dynamics of the system are then defined in terms of system state propagation in time following a Chapman-Kolmogorov formulation. These unobserved variables are further observed through the measured responses (e.g. acceleration, strain, etc.) employing a measurement model/equation involving uncertainties due to sensor noise. Although, the system dynamics can be better defined in the continuous time domain, to facilitate estimation using discretely sampled sensor measurements, both physical models are transformed into discrete time domain.

Depending on the nature of the formulated process and/or measurement model, several filter types have been proposed in the literature. For linear time invariant (LTI) systems (linear process and measurement model), Kalman filter (KF) can be identified as the most employed approach. On the introduction of non-linearity in either of the models (process and/or measurement) or time variability in the system, the inability of KF redirects to the usage of non-linear filter variants like Extended (EKF) 28, Unscented (UKF) [31, 37, Ensemble (EnKF) [22] KFs or Particle filter (PF) 24]. Non-linearity in the process model may also be caused due to non-linearity in the system itself; tensegrities being one such example manifesting geometric non-linearity. For linear/non-linear time variant (LTV/NLTV) systems, the system estimation is proceeded with first parameterizing the system and subsequently estimating them alongside as additional parameter states, $\boldsymbol{\theta}_{k}$. This, however, renders the assessed system to be non-linear due to the non-linear mapping of $\boldsymbol{\theta}_{k}$ to the corresponding measurements.

In the context of SHM, a set of location-based health indices (HIs) is employed for parameterizing the system health which are then estimated/monitored as the additional parameter states, $\boldsymbol{\theta}_{k}$. Estimation of the HIs can further be approached either jointly [36 or conditionally [17, 51 with respect to the real system states. The relative efficiency of the conditional over joint estimation approach has already been corroborated in several articles [13] and upon further introduction of interacting strategies by [32, the focus has strongly shifted to the use of individual filters for states or parameter estimation, like in Interacting Particle-Kalman filter (IPKF) [52, 71], Dual KF 9], Dual EKF (DEKF) [51, etc.

Within the context of tensegrity SHM, the self-stiffening property [49] can be accounted for by considering geometric non-linearity in the tensegrity dynamics [33. Eventually, with the non-linear tensegrity dynamics defined through this process model, the model is axiomatically non-linear. With $\boldsymbol{\theta}_{k}$ as additional states to be observed through measurements, the measurement model is also non-linear. Hence a major challenge in tensegrity SHM is to handle these non-linearities simultaneously and efficiently. PF has been successful in dealing with highly non-linear systems $[8,12,14$, although at the expense of high computational cost. To overcome the cost issue, IPKF was introduced 71 in which KF deals with the linear state estimation, while PF is employed for non-linear parameter estimation. Nevertheless, the dynamic model pertaining to tensegrity SHM is non-linear, invalidating the KF. The replacement can be chosen from the available filter variants. Of them, EnKF has been proved to be efficient in the propagation of non-linear system states [27. 
while allowing the entire health monitoring approach to be parallelized together with the PF. An Interacting Particle Ensemble Kalman Filter (IP-EnKF) has therefore been employed to estimate tensegrity health.

The algorithm has been formulated to make use of only strain gauge response as the measured data to the proposed IP-EnKF, since strain gauges are cheaper than accelerometers while being reported as more sensitive towards the presence of damage [50. Detailed discussion on the tensegrity modeling and simulating dynamic responses have been demonstrated in Section 2 with details of the state-space definition of the tensegrity dynamics (Section 2.2). The proposed IP-EnKF algorithm is further explained in Section 3 followed by a numerical validation study detailed in Section 4 that demonstrates the application of the proposed approach on a simplex tensegrity (ST) and an expanded-octahedron tensegrity (EOT) modules.

\section{Tensegrity model and dynamic response}

While modeling a tensegrity, suitable internal force inequalities should be added to the model to account for the nature of the dedicated tension cables or compression struts or bars that can take up both tension and compression forces, if present in the structure. This makes modeling of the tensegrities different from that of the typical truss structures. The design and identification of self-stressed configuration for tensegrities is a separate and much-explored field of research, not in the scope of this article. Yet for the sake of clarity, this article details the form-finding algorithm (Algorithm 2) employed in this study to identify the initial stable form of the tensegrity. Special measures are further taken to ensure that no local failure conditions (bar buckling and/or cable slacking) occur while finding the initial stable configuration of the tensegrities through constraining the member pre-stress levels.

To account for the large deformations of tensegrity members under external loading, geometric nonlinearity is introduced in the model. It has been observed that tensegrity with low pre-stress levels, manifests stronger non-linearity compared to tensegrities with higher pre-stress levels 40 . Consequent to load application and related changes in the configuration, the current strain-displacement relationship becomes an implicit problem involving the ever-evolving tensegrity configuration. With the finite element modeling (FEM) approach to discretize the spatial domain, the aspect of geometric non-linearity can be invoked without much complexity. Nevertheless, the implicit nature of the problem needs substantial computation within a recursive estimation approach which might render the involved SHM, although accurate, slow. Since with the Bayesian approach, the model inaccuracy can be complemented with recursive inferencing from the data, in this article an explicit representation of the strain-displacement relationship is adopted powered by explicit Newmark-beta method 15, 43. The modeling is detailed in the following.

\subsection{Geometric non-linear finite element model}

Modeling of tensegrity with a geometric non-linear FEM approach exists in literature 33. Except for geometric non-linearity, this article does not consider any other source of non-linearity, like material or 
boundary non-linearity. The initial form (involving coordinate position and member pre-stress levels) of the tensegrity is required to be identified through the form-finding approach following Algorithm 2 , Algorithm 2 presents a force-density based optimization approach for tensegrity form-finding that has been adopted to identify the initial coordinates and related pre-stress levels with constraints on the member pre-stress ensuring no tension/compression member is slacking/buckling, respectively. The resulting stable form and related data are presented in Figures 2 and 4 and Tables 1 and 2 respectively.

Next, at any arbitrary time instant $t$, for each of the $m^{t h}$ member/element of the self-stressed tensegrity, the associated global coordinates (defined in the global coordinate system (GCS), $x y z), \mathbf{q}^{m}(t)_{6 \times 1} \subset \mathbf{q}(t)$, are transformed to their counterparts, $\mathbf{q}^{m, l}(t)_{2 \times 1}$, in the local coordinate system (LCS), $\bar{x} \bar{y} \bar{z}$ (cf. Figure (1)), with the help of member-specific transformation matrix $\mathbf{T}^{m}(t)$. Here $\mathbf{q}(t)$ denotes the entire global coordinate set of all the tensegrity nodes.

$$
\mathbf{q}^{m, l}(t)=\mathbf{T}^{m}(t) \mathbf{q}^{m}(t)
$$

where, $\mathbf{T}^{m}(t)=\left[\begin{array}{cccccc}\cos \theta_{x}^{m}(t) & \cos \theta_{y}^{m}(t) & \cos \theta_{z}^{m}(t) & 0 & 0 & 0 \\ 0 & 0 & 0 & \cos \theta_{x}^{m}(t) & \cos \theta_{y}^{m}(t) & \cos \theta_{z}^{m}(t)\end{array}\right]$, $\mathbf{q}^{m, l}(t)=\left\{q_{1}^{l}(t) \quad q_{2}^{l}(t)\right\}^{m^{T}}$ and $\mathbf{q}^{m}(t)=\left\{\begin{array}{llllll}q_{1 x}(t) & q_{1 y}(t) & q_{1 z}(t) & q_{2 x}(t) & q_{2 y}(t) & q_{2 z}(t)\end{array}\right\}^{m^{T}}$. $\cos \theta_{x}^{m}(t), \cos \theta_{y}^{m}(t)$ and $\cos \theta_{z}^{m}(t)$ are time varying angular positions of the member $m$ with respect to GCS. A schematic for the assumed element is demonstrated in Figure 1.

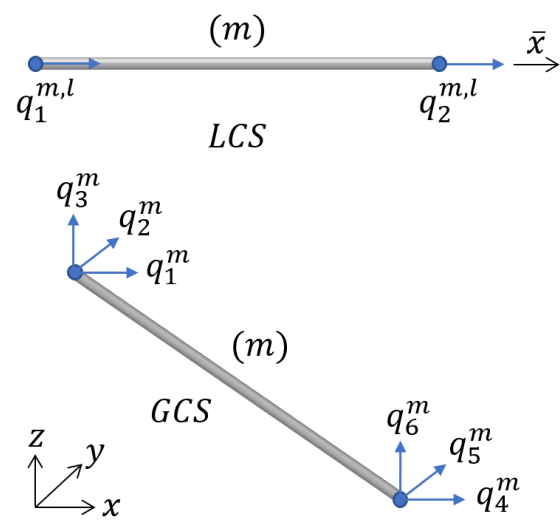

Figure 1: LCS and GCS for bar element type

The deformation, $\mathbf{u}^{m}(r, t)$, at any point within the element $m$ can further be described using shape functions $\left(N_{1}(r)\right.$ and $\left.N_{2}(\mathrm{r})\right)$ and local nodal displacements $\left(\mathbf{q}^{m, l}(t)\right)$.

$$
\mathbf{u}^{m}(r, t)=\left[N_{1}(r) \quad N_{2}(r)\right] \mathbf{q}^{m, l}(t)
$$

where the shape functions are described in natural coordinate system, $N_{1}(r)=(1-r) / 2$ and $N_{2}(r)=$ 
$(1+r) / 2$, for the ease of integration. $r$ being the natural variable defined within the range $-1 \leq r \leq 1$. To incorporate geometric non-linearity in strain, a second order relationship between Green's strain and displacement fields has been considered in this study (as per [33]),

$$
\varepsilon^{m}(r, t)=\frac{\partial \mathbf{u}^{m}(r, t)}{\partial x}+\frac{1}{2}\left(\frac{\partial \mathbf{u}^{m}(r, t)}{\partial x}\right)^{2}
$$

with $\mathbf{u}^{m}(r, t)$, as defined in Equation (2), the member strain field $\varepsilon^{m}(r, t)$ can further be expressed introducing linear $\left(\mathbf{B}_{L}^{m}\right)$ and non-linear $\left(\mathbf{B}_{N L}^{m}\right)$ strain-displacement matrices with $\mathbf{B}_{N L}^{m}\left(\mathbf{q}^{m, l}(t)\right)$ being a non-linear function of $\mathbf{q}^{m, l}(t)$. The functional representation of $\mathbf{B}_{N L}^{m}$ to demonstrate its dependence on the $\mathbf{q}^{m, l}(t)$ is although dropped from here on for the sake of compactness.

$$
\varepsilon^{m}(r, t)=\mathbf{B}_{L}^{m} \mathbf{q}^{m, l}(t)+\mathbf{B}_{N L}^{m} \mathbf{q}^{m, l}(t)
$$

$$
\text { where } \mathbf{B}_{L}^{m}=\left[\frac{\partial N_{1}(r)}{\partial x} \frac{\partial N_{2}(r)}{\partial x}\right] \text { and } \mathbf{B}_{N L}^{m}=\frac{1}{2} \mathbf{q}^{m, l}(t)^{T}\left[\begin{array}{l}
\partial N_{1}(r) / \partial x \\
\partial N_{2}(r) / \partial x
\end{array}\right]\left[\frac{\partial N_{1}(r)}{\partial x} \frac{\partial N_{2}(r)}{\partial x}\right] \text {. }
$$

The element tangent stiffness matrix can further be obtained by applying the principle of virtual work, i.e., minimizing the difference (i.e. virtual work, $\delta W$ ) between the work done by the internal forces (second Piola-Kirchhoff stress, $\left.\sigma^{m}(r, t)\right)$ undergoing incremental Green's strain $\delta \varepsilon^{m}(r, t)$ and the work done by the external forces undergoing virtual displacement $\delta \mathbf{q}^{m}(t)$ integrated over the entire volume, $V^{m}[29$. The virtual work can therefore be defined as,

$$
\delta W=\int_{V^{m}} \delta \varepsilon^{m}(r, t)^{T} \sigma^{m}(r, t) d V-\delta \mathbf{q}^{m}(t)^{T} \mathbf{F}(t)
$$

where, $\sigma^{m}(r, t)$ is obtained from the constitutive relation, $\sigma^{m}(r, t)=\mathbf{E}^{m} \varepsilon^{m}(r, t)$ with $\mathbf{E}^{m}$ being the constitutive matrix. The above equation is further expanded as follows,

$$
\delta W=\int_{V^{m}} \delta \mathbf{q}^{m}(t)^{T} \mathbf{T}^{m T} \mathbf{B}^{m T} \mathbf{E}^{m} \mathbf{B}^{m} \mathbf{T}^{m} \mathbf{q}^{m}(t) d V-\delta \mathbf{q}^{m}(t)^{T} \mathbf{F}(t)
$$

where, $\mathbf{B}^{m}=\mathbf{B}_{L}^{m}+\mathbf{B}_{N L}^{m}$, making $\mathbf{B}^{m}$ a function of $\mathbf{q}^{m, l}(t)$ as well. Further, ignoring the trivial part of the solution (i,e, $\delta \mathbf{q}^{m}(t) \neq 0$ ), and taking derivative of the internal force with respect to $\mathbf{q}^{m}(t)$, element tangential stiffness matrix $\mathbf{K}^{m}(t)$ can be defined in compact form as,

$$
\mathbf{K}^{m}(t)=\frac{A^{m} l^{m}}{2} \int_{-1}^{1} \frac{\partial\left(\mathbf{B}^{m T} \sigma^{m}(r, t)\right)}{\partial \mathbf{q}^{m}(t)} d r
$$

assuming a uniform cross section $A^{m}$ over the entire length $l^{m}$ of element $m$. Numerical integration of the above integral can be obtained through Gauss-Quadrature method with one Gauss-point. The tangential stiffness matrix $\left(\mathbf{K}^{m}(t)\right)$ can further be splitted into material $\left(\mathbf{K}_{M}^{m}(t)\right)$, geometric $\left(\mathbf{K}_{G}^{m}(t)\right)$ and initial displacement $\left(\mathbf{K}_{U}^{m}(t)\right)$ stiffness matrices [29, 60]:

$$
\mathbf{K}^{m}(t)=\mathbf{K}_{M}^{m}(t)+\mathbf{K}_{G}^{m}(t)+\mathbf{K}_{U}^{m}(t)
$$


where, $\mathbf{K}_{M}^{m}(t), \mathbf{K}_{G}^{m}(t)$ and $\mathbf{K}_{U}^{m}(t)$ are given by Equations $(9), 10$ and (11), respectively

$$
\begin{gathered}
\mathbf{K}_{M}^{m}(t)=\frac{\mathbf{E}^{m} A^{m} l^{m}}{2} \int_{-1}^{1} \mathbf{T}^{m T} \mathbf{B}_{L}^{m T} \mathbf{B}_{L}^{m} \mathbf{T}^{m} d r \\
\mathbf{K}_{G}^{m}(t)=\frac{A^{m} l^{m}}{2} \int_{-1}^{1} \frac{\partial \mathbf{B}_{N L}^{m}{ }^{T}}{\partial \mathbf{q}^{m}(t)} \sigma^{m}(r, t) d r \\
\mathbf{K}_{U}^{m}(t)=\frac{\mathbf{E}^{m} A^{m} l^{m}}{2} \int_{-1}^{1} \mathbf{T}^{m T}\left(\mathbf{B}_{L}^{m T} \mathbf{B}_{N L}^{m}+\mathbf{B}_{N L}^{m}{ }^{T} \mathbf{B}_{L}^{m}+\mathbf{B}_{N L}^{m}{ }^{T} \mathbf{B}_{N L}^{m}\right) \mathbf{T}^{m} d r
\end{gathered}
$$

Further, global tangential stiffness matrix $\mathbf{K}(t)$ can be obtained by assembling the elemental stiffness matrices and applying natural boundary conditions. Similarly, the mass matrix $\mathbf{M}$ can be obtained by following the consistent mass matrix assumption. The global tangent stiffness matrix $\mathbf{K}(t)$ is determined taking basis on updated Lagrange formulation that defines the stiffness at current time. For that, the initial displacement matrix has been recursively re-calibrated taking displacements from the last step.

\subsection{State space formulation of tensegrity dynamics}

Dynamics of typical truss structures can be defined with a linear second-order governing differential equation $(g d e)$. However, the embedded geometric non-linearity in the tensegrity model requires the dynamics to be defined using non-linear gde as,

$$
\mathbf{M} \ddot{\mathbf{q}}(t)+\mathbf{C}(t) \dot{\mathbf{q}}(t)+\mathbf{P}(\mathbf{q}(t))=\mathbf{F}(t)
$$

Clearly, the inelastic resisting force, $\mathbf{P}(\mathbf{q}(t))$, is non-linear and time-dependent due to the consideration of non-linear geometry. Specific to the tensegrity SHM problems under consideration, time dependency in $\mathbf{P}(\mathbf{q}(t))$ is also due to the varying health condition of the tensegrity. Suitable damping model for tensegrity is a well researched topic [58, 59] weighing the proportional and non-proportional damping models as options. It has been perceived in general, that compared to non-proportional damping models, proportional damping models are computationally inexpensive [1], although may lack accuracy sometimes [58]. The relative modeling inaccuracies can however be complemented with recursive Bayesian estimation approach in which the additional process noise can take care of this modeling uncertainty while benefiting the algorithm with promptness. Rayleigh damping has therefore been assumed for this tensegrity simulation. This is a classical viscous damping model assuming damping to be linearly proportional to mass and stiffness, as $\mathbf{C}(t)=a_{0}(t) \mathbf{M}+a_{1}(t) \mathbf{K}(t)$ where $\mathbf{K}(t)$ is the locally linearized tangent stiffness matrix. Although classical approach assumes the damping to be constant all through out, for non-linear systems with varying tangent stiffness matrix, updated stiffness is suggested to be employed along with varying proportionality coefficients (i.e. $a_{0}(t)$ and $\left.a_{1}(t)\right)$ instead of initial stiffness matrix [16, 30, 45. Further assumptions are imposed on first two modes being equally damped in order to estimate time varying coefficients $a_{0}(t)$ and $a_{1}(t)$. The details 
of Rayleigh damping model can be found in [15. Eventually, damping force being defined using Rayleigh's damping model, is also time dependent. Nevertheless, any other damping model can also be used instead 46].

The mass matrix is, however, considered to be time invariant. The structure is subjected to externally applied ambient forcing $\mathbf{F}(t)$ which is assumed to be not known explicitly, yet can be modeled as zero mean white Gaussian noise (WGN) of known stationary statistics $\mathbf{Q}$, as $\mathbf{v}_{k} \sim \mathcal{N}(0, \mathbf{Q})$.

The system dynamics can further be defined with displacement $(\mathbf{q}(t))$, velocity $(\dot{\mathbf{q}}(t))$ and acceleration $(\ddot{\mathbf{q}}(t))$ as system states observed through a set of strain measurements, $\left\{\varepsilon_{k}^{m}\right\}$, sampled in discrete time from the strain gauges patched on to the surface of the bars at their midpoints $(r=0.5)$. $\varepsilon_{k}^{m}$ is the discrete counterpart corresponding to its continuous time entity, $\varepsilon^{m}(r, t)$, with $k$ being the time instant at which the strain is sampled. To accommodate such discrete measurement, the non-linear state transition function has to be defined in discrete time state space formulation as,

$$
\mathbf{x}_{k}=f\left(\mathbf{x}_{k-1}, \mathbf{M}, \mathbf{K}_{k}, \mathbf{C}_{k}, d t, \mathbf{v}_{k}\right), \text { where } \mathbf{v}_{k} \sim \mathcal{N}(0, \mathbf{Q})
$$

Here, $\mathbf{x}_{k}=\left[\begin{array}{lll}\mathbf{q}_{k} & \dot{\mathbf{q}}_{k} & \ddot{\mathbf{q}}_{k}\end{array}\right]^{T}$, i.e. the discrete definition of the system states evolving over the non-linear state propagation function $f(\bullet) . \mathbf{q}_{k}, \dot{\mathbf{q}}_{k}, \ddot{\mathbf{q}}_{k}, \mathbf{M}, \mathbf{K}_{k}, \mathbf{C}_{k}$ are the respective discrete quantities corresponding to their continuous definitions. $d t$ is the time step for discretization. $\mathbf{v}_{k}$ has additionally been incorporated to collectively account for the uncertainties originating from the unavoidable model inaccuracies and ambient WGN force, $\mathbf{P}_{k}$. This WGN model is assumed with constant covariance $\mathbf{Q}$, same as the variance of the ambient force. Subsequently, the measurement equation can be defined as,

$$
\varepsilon_{k}=\mathbf{H B}\left(\mathbf{x}_{k}\right)+\mathbf{w}_{k}, \text { where } \mathbf{w}_{k} \sim \mathcal{N}(0, \mathbf{R})
$$

where, $\mathbf{B}(\bullet)$ denotes the global non-linear strain-displacement relationship for all members with $\mathbf{x}_{k}$ being its argument. $\mathbf{B}\left(\mathbf{x}_{k}\right)$ is acting here as a non-linear measurement function to map the unobserved states $\mathbf{x}_{k}$ to the measurement space. $\varepsilon_{k}$ consists of all the recorded member strains i.e., $\varepsilon_{k}=\left\{\varepsilon_{k}^{m}, m \in m^{o}\right\}$, where $m^{o}$ is the measured subset of $\mathbf{m},\left(\mathbf{m}=\cup\left\{m^{o} ; m^{u}\right\}\right)$ that are instrumented with strain gauges at their midpoints. Naturally, $m^{u}$ denotes the unobserved subset of $\mathbf{m}$. Accordingly, $\mathbf{H}$ stands for the selection matrix that isolates the measured member strains from all of the predicted set. $\mathbf{w}_{k} \sim \mathcal{N}(0, \mathbf{R})$ accounts for the sensor noise modeled as WGN process of constant covariance $\mathbf{R}$.

For system simulation, Newmark-beta method has been employed in its explicit formulation. The method is proven to have acceptable accuracy with non-linear dynamic simulations [10, 15, 43]. This approach takes its basis on an incremental equilibrium equation corresponding to the original dynamic equation (cf. 
Equation (12) to solve for the discrete non-linear structural response variables, i.e., $\ddot{\mathbf{q}}_{k}, \dot{\mathbf{q}}_{k}$ and $\mathbf{q}_{k}$,

$$
\mathbf{M} \Delta \ddot{\mathbf{q}}_{k}+\mathbf{C}_{k} \Delta \dot{\mathbf{q}}_{k}+\mathbf{K}_{k} \Delta \mathbf{q}_{k}=\Delta \mathbf{F}_{k}
$$

Operator $\Delta$ denotes the corresponding increment over each time step. Due to the non-linear geometry, the incremental equation is by nature implicit, for which iterative approach has to be adopted for accurate solution. Although, without compromising the accuracy by a substantial extent, Equation (15) can be solved using explicit formulation of Newmark-beta algorithm, detailed in Appendix B. This in turn facilitates with improved promptness of the damage detection by reducing computation in state propagation. Further, the method shows an unconditional stability for average constant acceleration assumption with $\gamma=0.5$ and $\beta=0.25$, as adopted in this article.

\subsection{Non-linearity in tensegrity dynamics}

In the following, the non-linearity of a tensegrity is investigated. For this, an EOT type tensegrity module has been selected (cf. Figure 2). The nodal positions, elemental connectivity and initial tension coefficients are presented in Table 1. For the simulation, the bars are assumed to act as compression as well as tension members, while cables take up only tension. The member connections are idealized as friction-less pin-joints. The assumptions made for the simulations are further presented here for lucid comprehension.

1. Members are connected by friction-less pin-joints.

2. Bars act as compression as well as tension members, while cables take up only tension.

3. Only geometric non-linearity is considered for the modeling.

4. In line with [70 the considered tensegrities are assumed to be constrained at certain nodes to a fixed base which minimizes the flexibility of bars due to Coriolis effect hence the Coriolis terms can be neglected in Equation 12 .

5. Newmark-beta algorithm assumes average acceleration method which is known to be unconditionally stable.

6. Rayleigh's proportional damping model is used to model damping in tensegrity.

The tensegrity is excited with a sinusoidal force $(=750 \sin (4 t) N)$ at its $3^{r d}$ node in x-direction. The related hysteresis and phase plane diagram curves are plotted in Figure 3. The hysteresis plot proves the existence of the non-linear relationship between displacement (as output) and forces (as input). For the phase plane diagram, displacement and velocity response of third node at its $\mathrm{x}$ dof is plotted. It is evident from the figures $3 \mathrm{a}$ and $3 \mathrm{~b}$ that the simulated tensegrity dynamics is demonstrating a non-linear behaviour. The phase plane diagram also establishes the dynamic stability of the assumed tensegrity under all assumed specifications of the tensegrity simulation. 


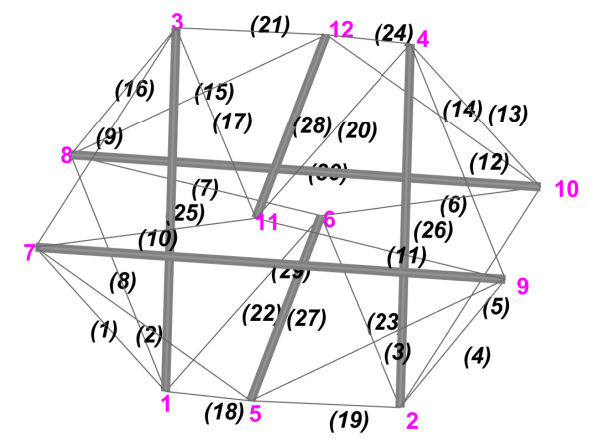

Figure 2: Expanded-octahedron tensegrity (EOT) configuration

Table 1: Nodal coordinates, elemental connectivity and initial tension coefficients of expanded-octahedron tensegrity (EOT) (with c:cable and b:bar)

\begin{tabular}{|c|c|c|c|c|c|c|c|c|c|c|c|c|c|}
\hline & Node & 1 & 2 & 3 & 4 & 5 & 6 & 7 & 8 & 9 & 10 & 11 & 12 \\
\hline \multirow{3}{*}{ EOT } & $\mathbf{X}$ & 0 & 0 & 0.548 & 0.548 & -1.726 & 2 & -0.658 & 1.205 & -0.657 & 1.205 & -1.452 & 2.274 \\
\hline & $\mathbf{Y}$ & 1 & -1 & 0.999 & -0.999 & 0 & 0 & 1.999 & 1.999 & -1.999 & -1.999 & $\overline{0}$ & $\overline{0}$ \\
\hline & $\mathbf{Z}$ & -2 & -2 & 0.904 & 0.904 & -1.548 & -1 & -0.685 & -0.411 & -0.685 & -0.411 & -0.096 & 0.45 \\
\hline
\end{tabular}

\begin{tabular}{|c|c|c|c|c|c|c|c|c|c|c|c|c|c|c|c|c|}
\hline & Element & 1 & 2 & 3 & 4 & $\mathbf{5}$ & 6 & 7 & 8 & 9 & 10 & 11 & 12 & 13 & 14 & 15 \\
\hline \multirow{9}{*}{ EOT } & Node 1 & 1 & 5 & 5 & 2 & 2 & 6 & 6 & 1 & 3 & 7 & 9 & 4 & 4 & 10 & 8 \\
\hline & Node 2 & 7 & 7 & 9 & 9 & 10 & 10 & 8 & 8 & 7 & 11 & 11 & 9 & 10 & 12 & 12 \\
\hline & Type & $c$ & $c$ & $c$ & $c$ & $c$ & $c$ & $c$ & $c$ & $c$ & $c$ & $c$ & $c$ & $c$ & $c$ & $c$ \\
\hline & Initial tension coeff. (N/m) & \multicolumn{15}{|c|}{0.8782} \\
\hline & & 16 & 17 & 18 & 19 & 20 & 21 & 22 & 23 & 24 & 25 & 26 & 27 & 28 & 29 & 30 \\
\hline & Node 1 & 3 & 3 & 1 & 2 & 4 & 3 & 1 & 2 & 4 & 1 & 2 & 5 & 11 & 7 & 8 \\
\hline & Node 2 & 8 & 11 & 5 & 5 & 11 & 12 & 6 & 6 & 12 & 3 & 4 & 6 & 12 & 9 & 10 \\
\hline & Type & $c$ & $c$ & $c$ & $c$ & $c$ & $c$ & $c$ & $c$ & $c$ & $b$ & $b$ & $b$ & $b$ & $b$ & $b$ \\
\hline & Initial tension coeff. $(\mathrm{N} / \mathrm{m})$ & \multicolumn{9}{|c|}{0.8782} & \multicolumn{6}{|c|}{-1.3173} \\
\hline
\end{tabular}

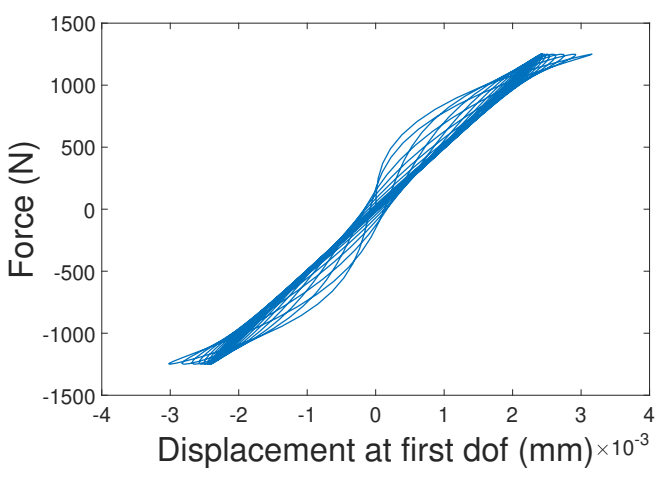

(a) Tensegrity hysteresis for EOT

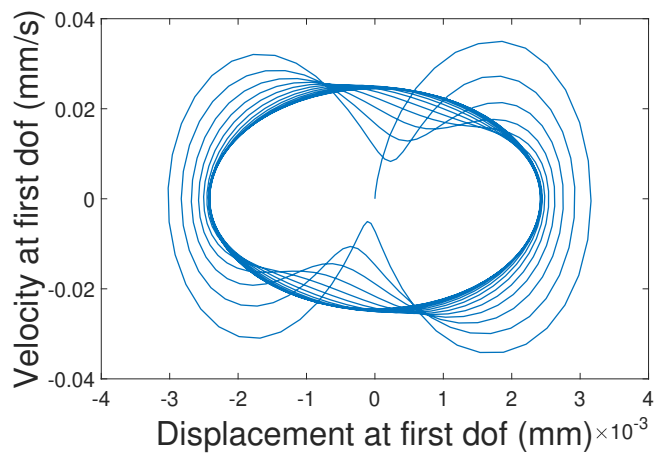

(b) Velocity - displacement plot for EOT

Figure 3: Non-linear behaviour of tensegrity system - EOT

\section{Proposed approach}

The system equation and simulation approaches for tensegrity structures have been demonstrated in Section 2. The approach for tensegrity SHM will further be detailed in this section. An IP-EnKF approach has been adopted for this in which the PF approaches the non-linear health parameter estimation while the EnKF estimates the non-linearly evolving system states $\mathbf{x}_{k}$ (as per Equation (13)). IP-EnKF can therefore be considered as an improvisation of IPKF 52 , wherein EnKF replaces KF to extend the reach of the 
algorithm to non-linear systems. It further facilitates with the option to parallelize the entire computation. The major aspect of this approach is that with IP-EnKF, compute-intensive PF handles only the severely non-linear parameter estimation problem while for the rest of the non-linear estimation, EnKF is employed. The pertinent interacting strategy between these two filter types is demonstrated later in this section.

For quantifying the health of the tensegrity, a set of location-based health indices (HIs) are devised. These HIs track the health of each individual members by a value within the range of 1 and 0 with 1 denoting healthy and 0 signifying completely damaged conditions. These time varying HIs are estimated with a vector $\boldsymbol{\theta}_{k}$ parameterizing the process model.

Provided that the process model of the system is known (at least as a sufficiently accurate model), error in the predicted output can be attributed to incorrect estimate of the model parameters $\boldsymbol{\theta}_{k}$. In the context of system health estimation, reduction in model parameter estimates can in turn signify a change in structural stiffness. Typically, structural stiffness is defined by its material (elasticity, cross-section, etc.) and geometric (configuration, pre-stress, etc.) stiffnesses. With the proposed algorithm, geometric stiffness of tensegrity is taken care of by introducing geometric non-linearity in the finite element model. Eventually, the prediction error can be attributed to a possible change in the material stiffness. Hence, for modeling purpose, damage in the members can be replicated through reduction in their initial elasticity, $\mathbb{E}_{0}$, using health indices, $\boldsymbol{\theta}_{k}$ as:

$$
\mathbb{S}_{k}\left(\boldsymbol{\theta}_{k}\right)=<\mathbb{S}_{0} \cdot \boldsymbol{\theta}_{k}>
$$

where, $\mathbb{S}_{0}=\left[\left(\mathbf{E}_{0}^{1} A_{0}^{1}\right), \ldots,\left(\mathbf{E}_{0}^{m} A_{0}^{m}\right)\right]$ is the vector encompassing the initial axial stiffness of all the tensegrity members, $\left[S_{0}^{1}, S_{0}^{2}, \cdots S_{0}^{m}\right]$. The reduced axial stiffness $\mathbb{S}_{k}$ of all the members at time step $k$, is thus a function of the health parameters $\boldsymbol{\theta}_{k} \cdot \boldsymbol{\theta}_{k}$, therefore, traces the alteration in the material stiffness of all the members of tensegrity, thereby detecting damage.

At any arbitrary time step $k$, PF propagates a set of $N_{p}$ parameter particles, $\boldsymbol{\Xi}_{k}=\left[\boldsymbol{\xi}_{k}^{1}, \boldsymbol{\xi}_{k}^{2}, \cdots, \boldsymbol{\xi}_{k}^{N_{p}}\right]_{m_{s} \times N_{p}}$, in time as realizations of the random variable $\boldsymbol{\theta}_{k}$. Each $j^{\text {th }}$ particle, $\boldsymbol{\xi}_{k}^{j}$, lists $m_{s} \times 1$ individual parameter realizations for HIs corresponding to $m_{s}$ members being monitored. This numerical approximation helps avoiding an explicit analytical integration over the entire parameter space, $\boldsymbol{\theta}_{k}$. The adopted particle evolution in time is basically a Gaussian perturbation around the current estimate of the particle $\boldsymbol{\xi}_{k-1}^{j}$,

$$
\boldsymbol{\xi}_{k}^{j}=\alpha \boldsymbol{\xi}_{k-1}^{j}+\mathcal{N}\left(\delta \boldsymbol{\xi}_{k} ; \boldsymbol{\sigma}_{k}^{\boldsymbol{\xi}}\right)
$$

where a Gaussian blurring is performed on $\boldsymbol{\xi}_{k-1}^{j}$ with a shift $\delta \boldsymbol{\xi}_{k}=(1-\alpha) \overline{\boldsymbol{\xi}}_{k-1}$ and a spread of $\boldsymbol{\sigma}_{k}^{\boldsymbol{\xi}}$. $\alpha$ is a hyper-parameter that controls the turbulence in the estimation. Upon prediction for the particle estimate in current time, the correction is performed according to the likelihood of the particle estimates against the measured data, detailed next. 
Eventually, the evolution of the parameter particles is automated and conditioned on their likelihood against measurement only, avoiding the requirement of any specific initial distribution for the particle space. To estimate the likelihood, the propagated particles are further put through the nested EnKF for state estimation. Within EnKF, $N_{e}$ state ensembles are propagated through the system (cf. Equation (13)). For this, current estimate for the stiffness matrix $\mathbf{K}_{k}$ is required. As per the current tensegrity configuration, extracted from the current estimates for the state ensembles, $\mathbf{x}_{k-1 \mid k-1}^{i, j}$, the member lengths, $l^{m}$, and transformation matrices, $\mathbf{T}^{m}$, are updated. Next, with current parameter particles, $\boldsymbol{\xi}_{k}^{j}$, and state ensembles, $\mathbf{x}_{k-1 \mid k-1}^{i, j}$, the current estimate for stiffness matrix, $\mathbf{K}_{k \mid k-1}^{i, j}$, is calculated. $\mathbf{K}_{k \mid k-1}^{i, j}$ is associated to $i^{\text {th }}$ ensemble, $\mathbf{x}_{k-1 \mid k-1}^{i, j}$, and $j^{t h}$ particle, $\boldsymbol{\xi}_{k}^{j}$. Thus combining Equations (7) and (16), the current estimate for $\mathbf{K}_{k \mid k-1}^{i, j}$ can be obtained as,

$$
\mathbf{K}_{k \mid k-1}^{i, j}=\mathcal{M}\left(\boldsymbol{\xi}_{k}^{j}, \mathbf{x}_{k-1 \mid k-1}^{i, j}\right)
$$

where, $\mathcal{M}(\bullet)$ is the stiffness calibration function that takes basis on the current tensegrity configuration. The prior state ensembles $\mathbf{x}_{k-1 \mid k-1}^{i, j}$ are further propagated to the next time step as propagated ensembles, $\mathbf{x}_{k \mid k-1}^{i, j}$, as per Equation (13). Subsequently, these propagated ensembles are observed as measurement predictions, $\mathbf{y}_{k \mid k-1}^{i, j}$, following Equation 14 . The process and measurement equation for the system is presented in the following.

$$
\begin{aligned}
& \mathbf{x}_{k \mid k-1}^{i, j}=f\left(\mathbf{x}_{k-1 \mid k-1}^{i, j}, \mathbf{K}_{k \mid k-1}^{i, j}, \mathbf{M}, d t, \mathbf{v}_{k}^{i, j}\right), \text { where } \mathbf{v}_{k}^{i, j} \sim \mathcal{N}(0, \mathbf{Q}) \\
& \mathbf{y}_{k \mid k-1}^{i, j}=\mathbf{H B}\left(\mathbf{x}_{k \mid k-1}^{i, j}\right)+\mathbf{w}_{k}^{i, j}, \text { where } \mathbf{w}_{k}^{i, j} \sim \mathcal{N}(0, \mathbf{R})
\end{aligned}
$$

Next, the predicted measurement, $\mathbf{y}_{k \mid k-1}^{i, j}$, is compared with the actual measurement obtained from the sensors. Innovation $\epsilon_{k}^{i, j}$ can be obtained as the deviation of $\mathbf{y}_{k \mid k-1}^{i, j}$ from the corresponding actual measurements $\mathbf{y}_{k}$. The innovation statistics is further quantified with an ensemble innovation mean $\epsilon_{k}^{j}=$ $\frac{1}{N_{e}} \sum_{i=1}^{N_{e}} \epsilon_{k}^{i, j}$. Next, the ensemble mean of propagated state estimates, $\mathbf{x}_{k \mid k-1}^{j}$, and predicted measurements, $\mathbf{y}_{k \mid k-1}^{j}$, are obtained as $\mathbf{x}_{k \mid k-1}^{j}=\frac{1}{N_{e}} \sum_{i=1}^{N_{e}} \mathbf{x}_{k \mid k-1}^{i, j}$ and $\mathbf{y}_{k \mid k-1}^{j}=\frac{1}{N_{e}} \sum_{i=1}^{N_{e}} \mathbf{y}_{k \mid k-1}^{i, j}$, respectively. Crosscovariance between state and measurement prediction, $C_{k}^{j, x y}$, and the measurement prediction covariance, $C_{k}^{j, y y}$, can further be computed as per [18].

$$
\begin{aligned}
C_{k}^{j, \mathbf{x y}} & =\frac{1}{N_{e}-1} \sum_{i=1}^{N_{e}}\left(\mathbf{x}_{k \mid k-1}^{j}-\mathbf{x}_{k \mid k-1}^{i, j}\right)\left(\mathbf{y}_{k \mid k-1}^{j}-\mathbf{y}_{k \mid k-1}^{i, j}\right)^{T} \\
C_{k}^{j, \mathbf{y}} & =\frac{1}{N_{e}-1} \sum_{i=1}^{N_{e}}\left(\mathbf{y}_{k \mid k-1}^{j}-\mathbf{y}_{k \mid k-1}^{i, j}\right)\left(\mathbf{y}_{k \mid k-1}^{j}-\mathbf{y}_{k \mid k-1}^{i, j}\right)^{T}
\end{aligned}
$$

${ }_{344}$ The innovation error covariance, $\mathbf{S}_{k}^{j}$, and EnKF gain, $\mathbf{G}_{k}^{j}$, are then obtained as $\mathbf{S}_{k}^{j}=C_{k}^{j, \mathbf{y y}}+\mathbf{R}$ and 
$\mathbf{G}_{k}^{j}=C_{k}^{j, \mathbf{x y}}\left(\mathbf{S}_{k}^{j}\right)^{-1}$. With this gain, the state ensembles are updated as,

$$
\mathbf{x}_{k \mid k}^{i, j}=\mathbf{x}_{k \mid k-1}^{i, j}+\mathbf{G}_{k}^{j} \epsilon_{k}^{i, j}
$$

The particle approximations for the states and parameters are then estimated as,

$$
\mathbf{x}_{k \mid k}=\sum_{j=1}^{N_{p}} w\left(\boldsymbol{\xi}_{k}^{j}\right) \mathbf{x}_{k \mid k}^{j} \quad \text { and } \quad \boldsymbol{\theta}_{k \mid k}=\sum_{j=1}^{N_{p}} w\left(\boldsymbol{\xi}_{k}^{j}\right) \boldsymbol{\xi}_{k}^{j}
$$

Finally, likelihood of each particle, i.e. $\mathcal{L}\left(\boldsymbol{\xi}_{k}^{j}\right)$, is calculated based on the innovation mean, $\epsilon_{k}^{j}$, and

$$
\mathcal{L}\left(\boldsymbol{\xi}_{k}^{j}\right)=\frac{1}{(2 \pi)^{n} \sqrt{\left|\mathbf{S}_{k}^{j}\right|}} e^{-0.5 \epsilon_{k}^{j T} \mathbf{S}_{k}^{j-1} \epsilon_{k}^{j}}
$$

The normalized weight for each $j^{t h}$ particle is further obtained using corresponding likelihood,

$$
w\left(\boldsymbol{\xi}_{k}^{j}\right)=\frac{w\left(\boldsymbol{\xi}_{k-1}^{j}\right) \mathcal{L}\left(\boldsymbol{\xi}_{k}^{j}\right)}{\sum_{j=1}^{N} w\left(\boldsymbol{\xi}_{k-1}^{j}\right) \mathcal{L}\left(\boldsymbol{\xi}_{k}^{j}\right)}
$$

For better understanding of the IP-EnKF algorithm used for tensegrity SHM, a pseudo code has been provided in Algorithm 1 .

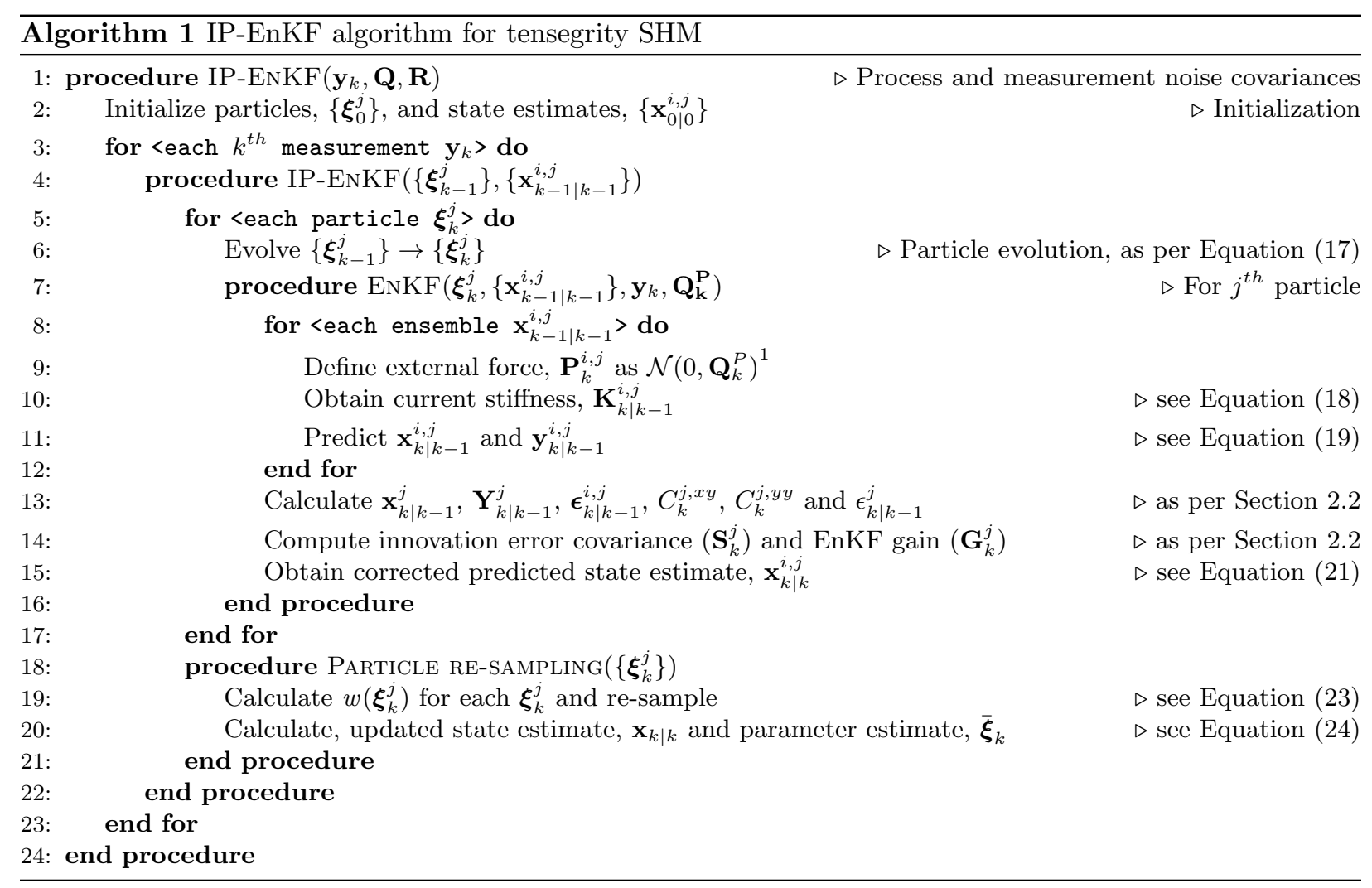




\section{Numerical Experiment}

Large scale tensegrity structures are typically designed or built as assemblage of several modular units as the basis of design and construction [47, 48. These modular units are connected to each other by tension mechanism (cables). To check the efficacy of the proposed algorithm for tensegrity SHM, it has been numerically tested on two of the most common tensegrity modules: simplex tensegrity (ST) and expandedoctahedron tensegrity (EOT). These modules are first numerically simulated for strain responses under a WGN forcing. However, prior to the numerical simulation, their initial forms are estimated following the process detailed in Algorithm 2.

In the following, a dynamic simulation is performed and strain data is collected from all the members that are not fixed. The responses from strain gauges are sampled at a fixed sampling frequency of $100 \mathrm{~Hz}$ [15, 55] for 5 seconds. Although average acceleration technique is unconditionally stable for all $d t$ values, the study used a $d t$ value that is also consistent with the explicit central difference scheme $(\omega \Delta t \leq 2)$. To mimic real-life sensor data, the computed strain data is contaminated by adding (1\%/2\%/5\%/10\%) SNR WGN. Henceforth, the contaminated strain data is used as the actual measured data, $\mathbf{y}_{k}$, for IP-EnKF algorithm that has been used for tensegrity SHM.

For both the aforementioned cases (ST and EOT), the effect of measurement noise level on damage detection accuracy along with the extent of damage that can be detected with precision, has been studied. The ability of the algorithm to detect multiple damages in a tensegrity has also been tested. The initial self-stressed configurations of tensegrity modules, ST (cf. Figure 4) and EOT (cf. Figure 2) have been obtained through force density-based form-finding algorithm $\sqrt{\text { Appendix A }}$ and are presented in Tables 2 and 1. respectively, in terms of self-stress coordinates, member connectivity and initial tension coefficients.

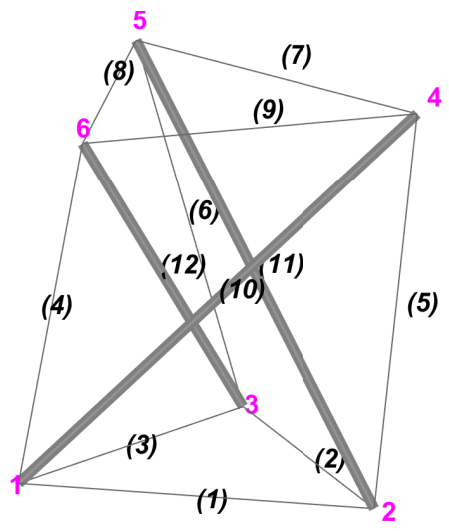

Figure 4: Simplex Tensegrity configuration

Adopted ST is a cylindrical tensegrity with 3 bars and 6 cables, whereas the EOT is a spherically symmetric tensegrity with 6 bars and 24 cables. The algorithm Appendix A to obtain initial statically 
stable coordinates has been verified with [7. All the members, cables and bars, of both the tensegrities are assumed to be made of steel (modulus of elasticity $=200 G P a)$. The diameters of bar and cable members are taken as $20 \mathrm{~mm}$ and $5 \mathrm{~mm}$, respectively. For dynamic analysis, each of them is connected to a fixed base at three nodes: (1-3) for ST (cf. Figure 4) and $(1,2,6)$ for EOT (cf. Figure 2). The stable form of the tensegrities is further excited with an ambient Gaussian force (elaborated later for each case) applied to ST and EOT on the fourth and the third node, respectively, in x-direction.

Table 2: Nodal coordinates, elemental connectivity and initial tension coefficients of simplex tensegrity (ST) (with c:cable and b:bar)

\begin{tabular}{|c|c|c|c|c|c|c|c|}
\hline & Node & $\mathbf{1}$ & $\mathbf{2}$ & $\mathbf{3}$ & $\mathbf{4}$ & $\mathbf{5}$ & $\mathbf{6}$ \\
\hline \multirow{3}{*}{ ST } & $\mathbf{X}$ & 0.577 & -0.244 & -0.266 & -0.452 & 0.0094 & 0.509 \\
\cline { 2 - 8 } & $\mathbf{Y}$ & 0 & 0.5 & -0.461 & 0.301 & -0.542 & 0.279 \\
\cline { 2 - 8 } & $\mathbf{Z}$ & 0 & 0 & 0 & 0.919 & 0.919 & 0.919 \\
\hline
\end{tabular}

\begin{tabular}{|c|c|c|c|c|c|c|c|c|c|c|c|c|c|}
\hline & Element & $\mathbf{1}$ & $\mathbf{2}$ & $\mathbf{3}$ & $\mathbf{4}$ & $\mathbf{5}$ & $\mathbf{6}$ & $\mathbf{7}$ & $\mathbf{8}$ & $\mathbf{9}$ & $\mathbf{1 0}$ & $\mathbf{1 1}$ & $\mathbf{1 2}$ \\
\hline \multirow{4}{*}{ ST } & 1 & 2 & 3 & 1 & 2 & 3 & 4 & 5 & 6 & 1 & 2 & 3 \\
\cline { 2 - 13 } & Node 1 & 2 & 3 & 1 & 6 & 4 & 5 & 5 & 6 & 4 & 4 & 5 & 6 \\
\cline { 2 - 12 } & Node 2 & $c$ & $c$ & $c$ & $c$ & $c$ & $c$ & $c$ & $c$ & $c$ & $b$ & $b$ & $b$ \\
\cline { 2 - 11 } & Type & \multicolumn{2}{|c|}{0.6834} & \multicolumn{2}{|c|}{1.1837} & \multicolumn{2}{|c|}{0.6835} & \multicolumn{2}{c}{-1.1838} \\
\hline
\end{tabular}

For both the tensegrity modules, damage is induced in their members $0.5 \mathrm{~s}$ after the simulation starts. The initial distribution type for the parameter particles, $\boldsymbol{\theta}_{k}$ (HIs) is set to be Gaussian, with their mean set as 1 assuming an undamaged condition and a standard deviation of 0.02, with $\alpha$ chosen as 0.90 (cf. Equation 17p). For consistency and understanding, the HIs of damaged members are compared to the $\mathbf{H I}(=1)$ of undamaged member 10 for all the cases.

\subsection{Effect of external load on vibrational properties of undamaged tensegrity}

As already discussed in the article, upon load application, the vibrational properties of a tensegrity change due to a change in the tensegrity stiffness owing to the change in the pre-stress. This has been demonstrated through an example case study on the ST subjected to an external WGN load of variance $1.25 \times 10^{4} N^{2}$. In the case study, the WGN is applied on the fourth node along its x-direction (cf. Figure 44. No member is damaged and the system is simulated for 5 seconds. The responses are recorded at a sampling frequency of $100 \mathrm{~Hz}$. It is observed that under varying external load, natural frequencies of ST change considerably even in the absence of any damage. Figure 5 demonstrates the relative change in first three natural frequencies $\left(\omega_{1}, \omega_{2}\right.$ and $\left.\omega_{3}\right)$ in time under a time varying load in comparison to their values $\left(\omega_{1}^{0}, \omega_{2}^{0}\right.$ and $\left.\omega_{3}^{0}\right)$ corresponding to a stable form. Clearly, this establishes that modal comparison is not an option for tensegrity SHM and establishes the necessity for time domain approaches. Further, since the tensegrity stiffness is a function of force, a tensegrity with unknown force can not evidently be estimated with a deterministic approach. This emphasizes the need for probabilistic approaches in which the system health can be estimated with a probabilistic measure and thereby justifies the employment of the proposed Bayesian filtering-based algorithm. 

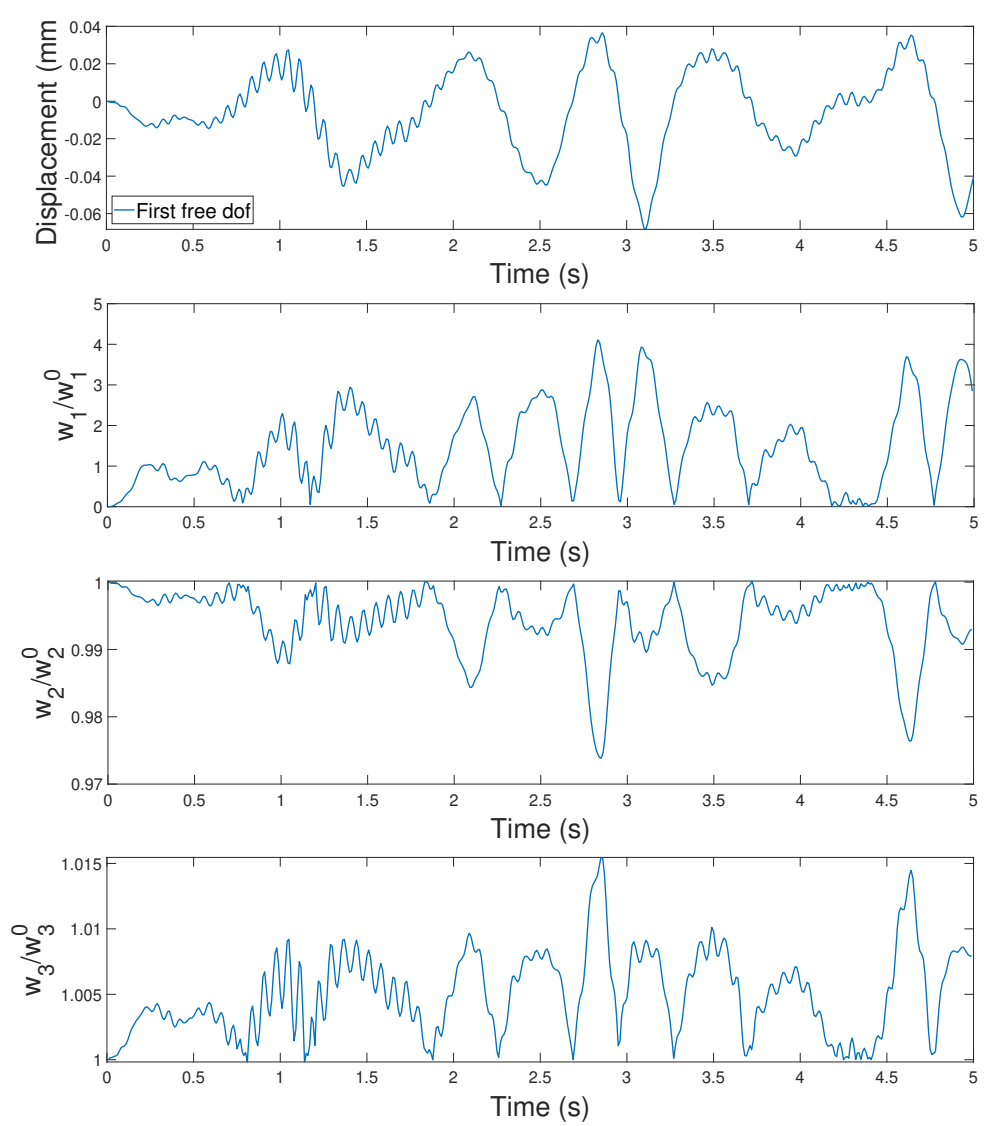

Figure 5: Variation in frequency $\left(1^{\text {st }}, 2^{\text {nd }} \& 3^{\text {rd }}\right.$ ) of simplex (undamaged) under varying load

\subsection{Calibration of particle and ensemble pool}

A calibration study has been performed on ST to identify the minimum number of particles and ensembles that can be utilized to efficiently identify the damage induced. The details of tensegrity configuration, force statistics as well as simulation specification have been kept the same as specified in section 4.1. Further a $90 \%$ damage is induced in the $11^{\text {th }}$ member of the simplex. A set of numerical experiments are further performed targeting evaluation of the optimal number of particles and ensembles to be utilized for the rest of the numerical experiments based on the algorithm's performance for accuracy and computational time.

Firstly, the number of particles are varied as 500, 1000, 2500 and 5000 for an ensemble pool size of 50 ensembles (cf. Figure 6a). It has been observed that, beyond a particle pool size of 2500, the accuracy is not improving any further while only the computational expense is increasing substantially. Thus, a pool size of 2500 particles is chosen for PF. Next, optimal number of ensembles has been tested for EnKF with ensemble pool sizes of 75 and 100 (cf. Figure 6). Again it has been observed that an ensemble pool of 100 ensembles is sufficient to achieve desired accuracy while being within a manageable computational demand. A lower value of ensemble number (here, 50) decreases the overall accuracy of the algorithm for all particle sizes $(500 / 1000 / 2500 / 5000)$. It should also be noted that increasing the particle size improves the promptness 
in detection for the algorithm while increasing the computational cost of the algorithm as well. Thus for estimation of the tensegrity health with proposed IP-EnKF, 2500 filter particles are selected for the PF while 100 ensembles are chosen for the EnKF.

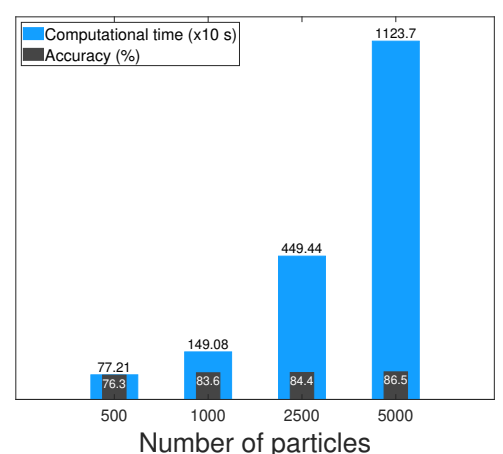

(a) $N_{E n s}=50$

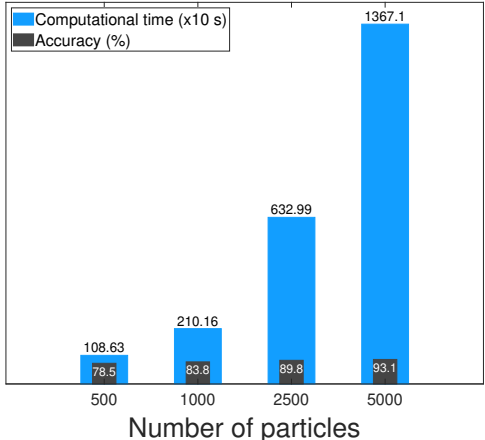

(b) $N_{E n s}=75$

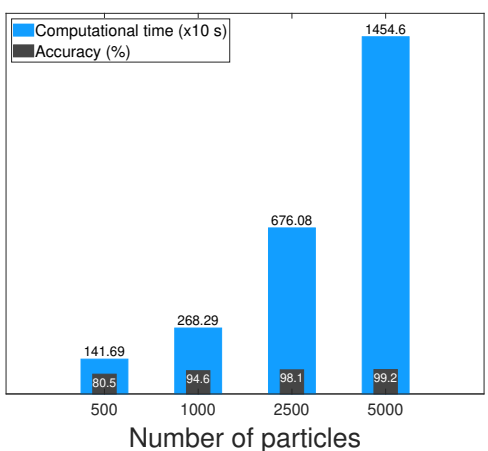

(c) $N_{\text {Ens }}=100$

Figure 6: Effect of number of particles and ensembles $\left(N_{E n s}\right)$ on accuracy and computational time of the algorithm

\subsection{Simplex tensegrity $(S T)$}

In the following, the proposed algorithm is tested on an ST module (cf. figure 4) while keeping the force statistics, application node, and other simulation specifications, the same as provided in section 4.1 for the sake of consistency. Again a damage is induced in its $11^{\text {th }}$ member (bar) by numerically reducing its stiffness by $90 \%, 0.5 \mathrm{~s}$ after the start of simulation. Strain measurements are collected from all the unrestricted members of ST, i.e, members $\{4-12\}$, under various SNR levels.

The proposed algorithm is tested for its sensitivity against measurement noise contamination. Four SNR levels are selected for this comparison: $1 \%, 2 \%, 5 \%$ and $10 \%$. Damages have been detected, localized and quantified for all noise levels (cf. Figure 7) with acceptable accuracy; although the promptness is not observed with noise of $10 \%$ SNR (cf. Figure 7). Clearly, this states that the proposed algorithm is sufficiently accurate with practical noise contamination levels.

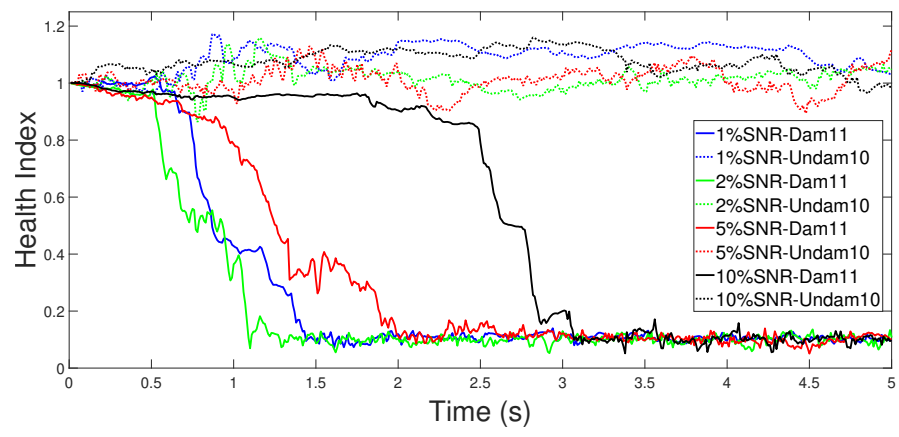

Figure 7: Measurement noise sensitivity of proposed approach - ST

To check the capability of the proposed approach to detect multiple damage in a tensegrity, a $90 \%$ 
damage $(\mathbf{H I}=0.1)$ is introduced to $8^{\text {th }}$ and $11^{\text {th }}$ member of the ST (cable and bar, respectively). The damage is induced simultaneously after $0.5 \mathrm{~s}$ of simulation. The simulated strain data is contaminated with 1\% SNR WGN. The algorithm is capable to detect multiple damages, irrespective of the type of member (cable or bar), with equal promptness and precision (cf. Figure 8).

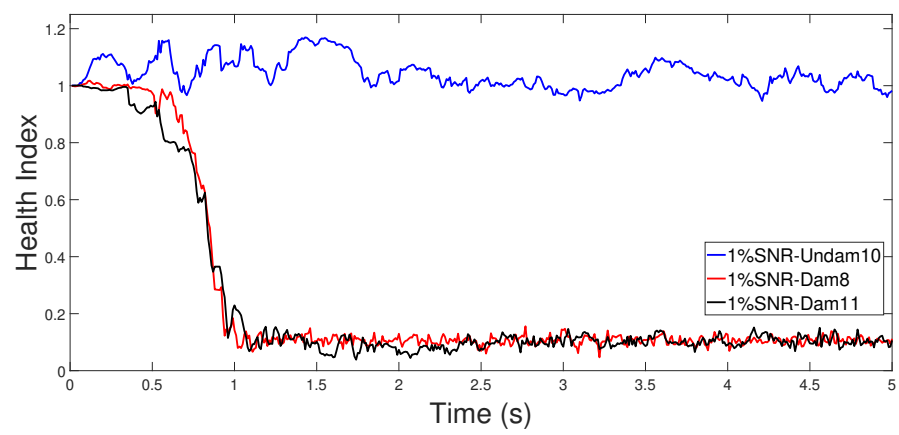

Figure 8: Multiple damage detection by the proposed approach - ST

Figure 9, shows the ability of the proposed approach to identify various damage levels $(10 \%, 20 \%, 30 \%$, $40 \%$ and $90 \%$ ) with corresponding $\mathbf{H I} \mathbf{s}=\{0.9,0.8,0.7,0.6$ and 0.1$\}$. It has been observed that for the lower damage levels $(10 \%)$, the algorithms output might confuse the investigator since the accuracy of the estimation may get masked within the estimation variation. However, for moderate or high levels of damage, demarcation of damaged state is quite straightforward with the proposed algorithm. It has been experienced that the proposed algorithm can effectively demarcate a damaged member having a damage level as small as $20 \%(\mathbf{H I}=0.8)$ without any confusion.

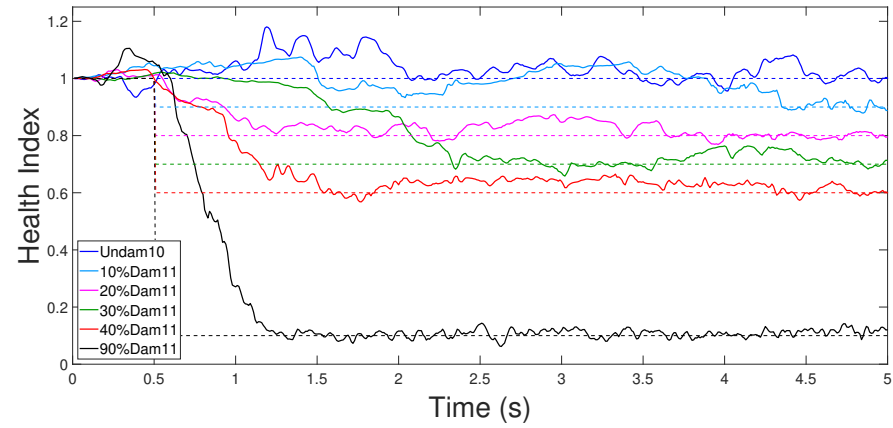

Figure 9: Detection of various damage levels by the proposed approach - ST

The minimum number of strain gauges required by the proposed approach to detect damage in ST, has been further investigated (cf. Figure 10). Following cases have been included, i) 9 strain gauges $\{4-12\}$,

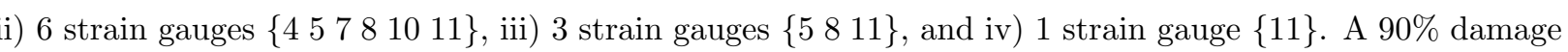
is induced in the $11^{\text {th }}$ member for all the above cases. It is observed that the algorithm is able to detect the damage with acceptable level of accuracy, even with a single strain gauge. Notably, the placement of sensor plays a major role in precision and promptness of the algorithm: sensors in the vicinity of the damages always 
alleviate the effort to detect them. This has been exhibited by the proposed approach as well. Nevertheless, with the increasing numbers of sensors, this problem is observed to attenuate. This aspect is however very much system specific. Accordingly, this case study can only give an idea about minimum sensors required and as such can not help to interpret the efficacy of the proposed algorithm.

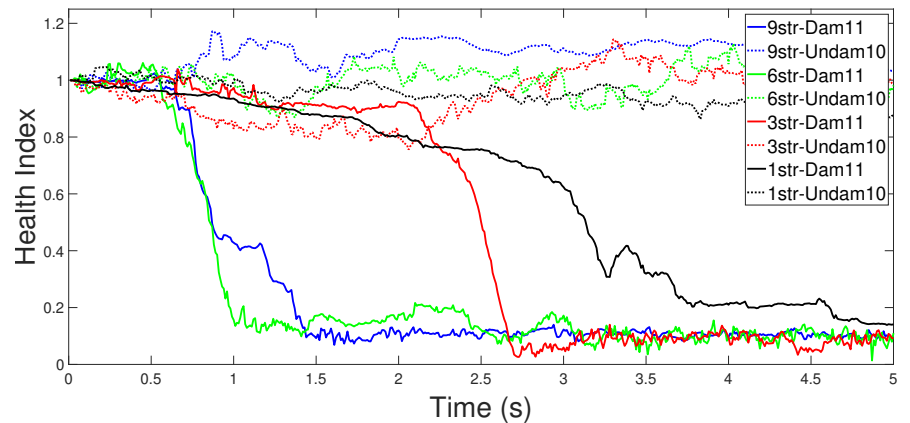

Figure 10: Performance of proposed approach under varying number of strain gauges - ST

\subsection{Expanded-octahedron tensegrity (EOT)}

Further, similar numerical experiments are performed on an EOT module, three times larger in dof s than the ST tested before. The objective is to check the efficacy of the algorithm with larger systems. An external WGN load of variance $1.25 \times 10^{4} N^{2}$ has been applied on the third node along its x-direction (cf. Figure 2). $0.5 \mathrm{~s}$ from the start of the simulation, a $90 \%$ damage level in the $11^{\text {th }}$ member (cable) of EOT is simulated. The sizes of particle and ensemble pool were selected as 2500 and 100, respectively. Strain measurements are collected from all the unrestricted members, i.e, members $\{1-21,24-30\}$ of the EOT, under various SNR (1\%,2\%, 5\% and 10\%). As was observed for ST, the estimation is found to be prompt and accurate till noise contamination level of 5\% SNR WGN (cf. Figure 11), beyond which (10\% SNR) promptness is compromised while estimation still being accurate. It has further been realized that for highly noisy systems, promptness can be regained by increasing the number of particles, ensembles or both, which however comes at a higher computational cost.

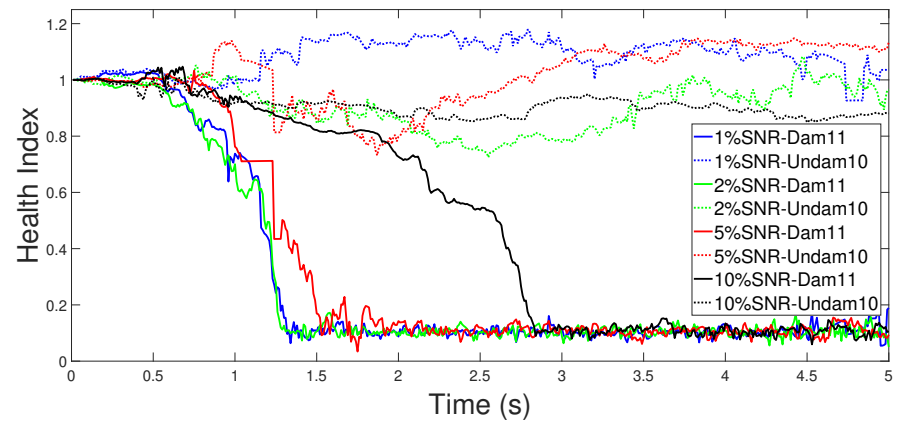

Figure 11: Measurement noise sensitivity of proposed approach - EOT 
To investigate the efficacy of the algorithm for multiple damage cases in EOT, two cable members (11 and 24) are simultaneously damaged to $90 \%$ damage level. A noise of $1 \%$ SNR level is added to the strain data. It has been observed (cf. Figure 12) that the algorithm is able to detect damage with equal precision and promptness, even if same member types (cables in this case) are damaged.

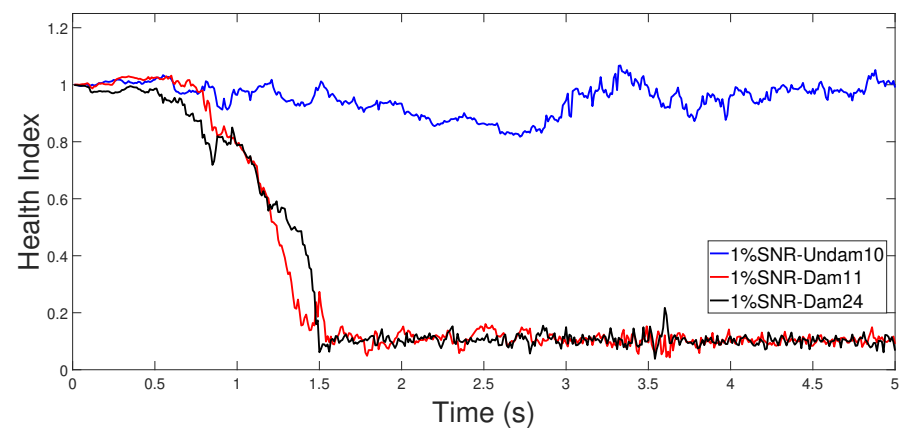

Figure 12: Multiple damage detection by the proposed approach - E0T

The algorithm is further tested to determine the extent of damage level that can be estimated for EOT (cf. Figure 13). Five different damage levels: 10\%, 20\%, 30\%, 40\% and 90\%, are tested in this endeavor. The algorithm precisely detects a damage level of $20 \%$, corresponding to an $\mathbf{H I}=0.8$.

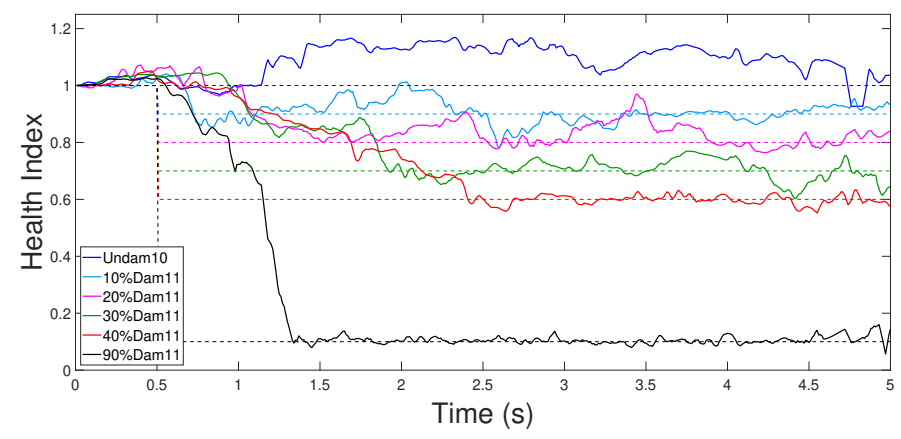

Figure 13: Detection of various damage levels by the proposed approach - EOT number as low as one (cf. Figure 14). The observation has been made by applying a $90 \%$ damage in the $11^{\text {th }}$ member of EOT, for each of the following cases, i) 28 strain gauges $\{1-21,24-30\}$, ii) 15 strain

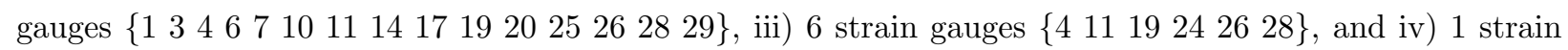
gauge $\{11\}$. A decrease in the employed number of sensors is observed to affect the promptness of detection. Further, a few false positives (for damages below 40\%) have also been observed for lower sensor number. This is although expected since compared to ST, EOT is defined with higher dof s and therefore needs more sensors to get monitored.

Finally, it has been observed that for both the tensegrities, the poor detection performance of the algorithm, owing to higher noise contamination and/or weaker damage levels, can still be improved by employing 


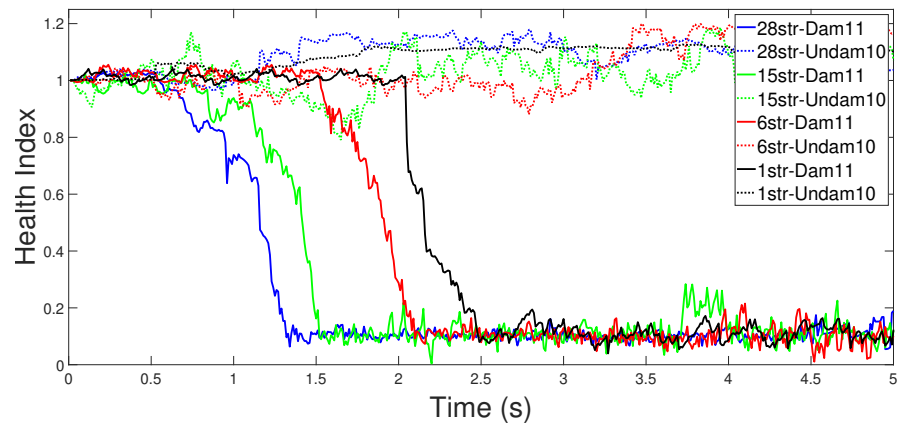

Figure 14: Performance of proposed approach under varying number of strain gauges - EOT

bigger particle and/or ensemble pools. Two sets of experiments are performed on ST and EOT specifically for those cases for which the algorithm performed poorly (i.e. cases with $10 \%$ SNR noise contamination and $10 \%$ damage). For both ST and EOT, the loss of promptness due to high level of noise contamination is regained (cf. 15a and 16a) after enhancing the particle and ensemble pools to 5000 and 200 respectively. The enhanced pool sizes also improved the precision and stability for the estimation of weak damages (cf. Figures $15 \mathrm{~b}$ and $16 \mathrm{~b}$. This in turn enables the algorithm to handle more complicated problems using compute-intensive approaches.

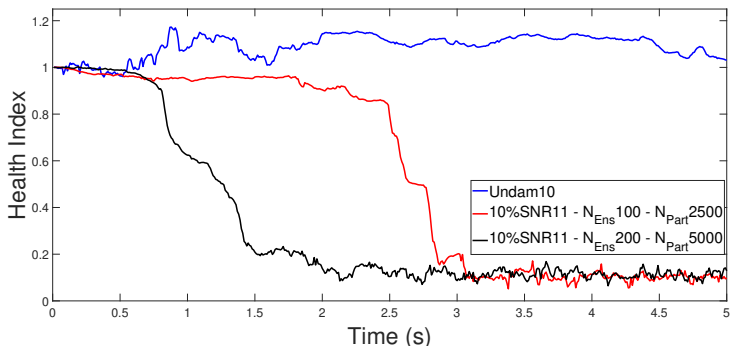

(a) Damage detection from a highly contaminated response

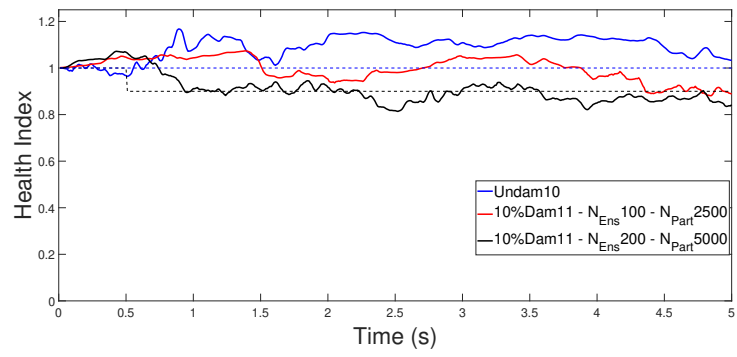

(b) Weak damage level detection

Figure 15: Effect of selecting a bigger particle and/or ensemble pools - ST

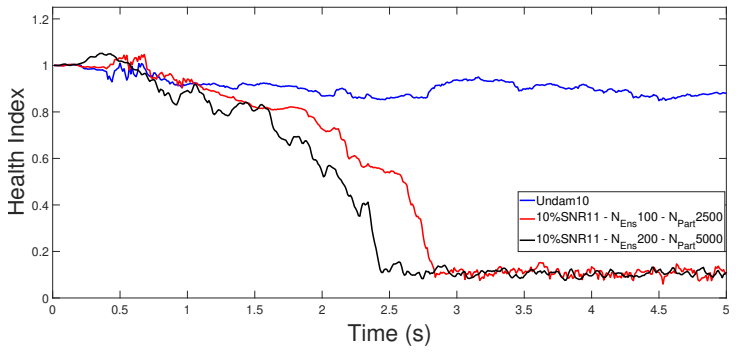

(a) Damage detection from a highly contaminated response

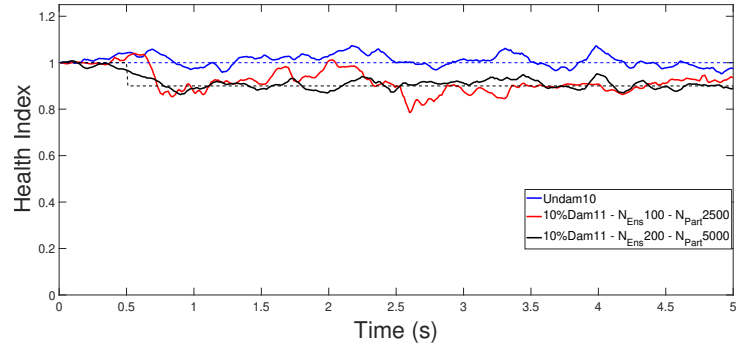

(b) Weak damage level detection

Figure 16: Effect of selecting a bigger particle and/or ensemble pools - EOT 


\section{Conclusion}

A novel interacting filtering based damage detection approach has been proposed for tensegrity structures. The approach successfully estimates the health parameters, through PF, along with the system states, through EnKF nested inside the PF. Proposed probabilistic approach enables monitoring the tensegrity health as long as a precise model of tensegrity dynamics is available and the input forcing statistics is known to the investigator. No explicit knowledge of input time history is required for the estimation. The method is found to be efficient in accurate detection and localization of the tensegrity damages and sufficiently robust against practical levels of measurement noise. The algorithm is observed to perform even with sparse instrumentation. Multiple damage cases were also detected without any confusion. Promptness and precision is observed to be affected for the weak damage cases and/or highly contaminated signals. Nevertheless, it has also been observed that the performance for such cases can be rectified by employing bigger particle and/or ensemble pools at a higher computational cost. The algorithm however restricts itself for tensegrities subjected to stationary Gaussian forcing only. Further research is required in order to develop tensegrity SHM approaches that are robust against input forcing.

Funding: This study was funded by Science \& Engineering Research Board (SERB), New Delhi, India, through grant file no. ECR/2018/001464.

\section{References}

[1] Adhikari, S., 2000. Damping models for structural vibration. Ph.D. thesis. Dissertation, University of Cambridge.

[2] Ali, N.B.H., Rhode-Barbarigos, L., Albi, A.A.P., Smith, I.F., 2010. Design optimization and dynamic analysis of a tensegrity-based footbridge. Engineering Structures 32, 3650-3659.

[3] Ashwear, N., Eriksson, A., 2014. Natural frequencies describe the pre-stress in tensegrity structures. Computers \& Structures 138, 162-171.

[4] Ashwear, N., Eriksson, A., 2017. Vibration health monitoring for tensegrity structures. Mechanical Systems and Signal Processing 85, 625-637.

[5] Aswal, N., Sen, S., 2020. Design and health monitoring of tensegrity structures: An overview, in: Reliability, Safety and Hazard Assessment for Risk-Based Technologies. Springer, pp. 523-533.

[6] Atig, M., El Ouni, M.H., Ben Kahla, N., 2019. Dynamic stability analysis of tensegrity systems. European Journal of Environmental and Civil Engineering 23, 675-692.

[7] Attig, M., Abdelghani, M., Kahla, N.b., 2016. Output-only modal identification of tensegrity structures. Engineering Structures and Technologies 8, 52-64.

[8] Azam, S.E., Bagherinia, M., Mariani, S., 2012. Stochastic system identification via particle and sigma-point kalman filtering. Scientia Iranica 19, 982-991.

[9] Azam, S.E., Chatzi, E., Papadimitriou, C., 2015. A dual kalman filter approach for state estimation via output-only acceleration measurements. Mechanical Systems and Signal Processing 60, 866-886.

[10] Ben Kahla, N., Moussa, B., Pons, J., 2000. Nonlinear dynamic analysis of tensegrity systems. Journal of The International Association for Shell and Spatial Structures 41, 49-58.

[11] Bhalla, S., Panigrahi, R., Gupta, A., 2013. Damage assessment of tensegrity structures using piezo transducers. Meccanica 48, 1465-1478.

[12] Chatzi, E.N., Smyth, A.W., 2009. The unscented kalman filter and particle filter methods for nonlinear structural system identification with non-collocated heterogeneous sensing. Structural Control and Health Monitoring: The Official Journal of the International Association for Structural Control and Monitoring and of the European Association for the Control of Structures 16, 99-123.

[13] Chen, Z., et al., 2003. Bayesian filtering: From kalman filters to particle filters, and beyond. Statistics 182 , 1-69.

[14] Ching, J., Beck, J.L., Porter, K.A., 2006. Bayesian state and parameter estimation of uncertain dynamical systems. Probabilistic engineering mechanics 21, 81-96.

[15] Chopra, A.K., 1995. Dynamics of structures, a primer. volume 2. Earthquake Engineering Research. 
[16] Chopra, A.K., McKenna, F., 2016. Modeling viscous damping in nonlinear response history analysis of buildings for earthquake excitation. Earthquake Engineering \& Structural Dynamics 45, 193-211.

[17] Doucet, A., De Freitas, N., Murphy, K., Russell, S., 2000. Rao-blackwellised particle filtering for dynamic bayesian networks, in: Proceedings of the Sixteenth conference on Uncertainty in artificial intelligence, Morgan Kaufmann Publishers Inc.. pp. 176-183.

[18] Evensen, G., 2003. The ensemble kalman filter: Theoretical formulation and practical implementation. Ocean dynamics 53, 343-367.

[19] Faroughi, S., Tur, J.M.M., 2015. Vibration properties in the design of tensegrity structure. Journal of Vibration and Control 21, 611-624.

[20] Feng, X., Ou, Y., Miah, M.S., 2018. Energy-based comparative analysis of optimal active control schemes for clustered tensegrity structures. Structural Control and Health Monitoring 25, e2215.

[21] Furuya, H., 1992. Concept of deployable tensegrity structures in space application. International Journal of Space Structures 7, 143-151.

[22] Ghanem, R., Ferro, G., 2006. Health monitoring for strongly non-linear systems using the ensemble kalman filter. Structural Control and Health Monitoring: The Official Journal of the International Association for Structural Control and Monitoring and of the European Association for the Control of Structures 13, 245-259.

[23] Gilewski, W., Kłosowska, J., Obara, P., 2015. Applications of tensegrity structures in civil engineering. Procedia Engineering 111, 242-248.

[24] Gordon, N.J., Salmond, D.J., Smith, A.F., 1993. Novel approach to nonlinear/non-gaussian bayesian state estimation, in: IEE proceedings $\mathrm{F}$ (radar and signal processing), IET. pp. 107-113.

[25] Hanaor, A., 1993. Double-layer tensegrity grids as deployable structures. International Journal of Space Structures 8, $135-143$.

[26] Hanaor, A., 2012. Debunking "tensegrity"-a personal perspective. International Journal of Space Structures 27, $179-183$.

[27] Hommels, A., Murakami, A., Nishimura, S.I., 2009. A comparison of the ensemble kalman filter with the unscented kalman filter: application to the construction of a road embankment. Geotechniek 13, 52 .

[28] Hoshiya, M., Saito, E., 1984. Structural identification by extended kalman filter. Journal of engineering mechanics 110, 1757-1770.

[29] Imai, K., Frangopol, D.M., 2000. Geometrically nonlinear finite element reliability analysis of structural systems. i: theory. Computers \& Structures 77, 677-691.

[30] Jehel, P., Léger, P., Ibrahimbegovic, A., 2014. Initial versus tangent stiffness-based rayleigh damping in inelastic time history seismic analyses. Earthquake Engineering \& Structural Dynamics 43, 467-484.

[31] Julier, S.J., Uhlmann, J.K., 1997. New extension of the kalman filter to nonlinear systems, in: Signal processing, sensor fusion, and target recognition VI, International Society for Optics and Photonics. pp. 182-194.

[32] Karlsson, R., Schon, T., Gustafsson, F., 2005. Complexity analysis of the marginalized particle filter. IEEE Transactions on Signal Processing 53, 4408-4411. doi 10.1109/TSP.2005.857061.

[33] Kebiche, K., Kazi-Aoual, M., Motro, R., 1999. Geometrical non-linear analysis of tensegrity systems. Engineering structures $21,864-876$.

[34] Levin, S.M., 2002. The tensegrity-truss as a model for spine mechanics: Biotensegrity. Journal of Mechanics in Medicine and Biology 02, 375-388. doi 10.1142/s0219519402000472.

[35] Li, T., Ma, Y., 2013. Robust vibration control of flexible tensegrity structure via $\mu$ synthesis. Structural control and health monitoring 20, 173-186.

[36] Lourens, E., Papadimitriou, C., Gillijns, S., Reynders, E., De Roeck, G., Lombaert, G., 2012. Joint input-response estimation for structural systems based on reduced-order models and vibration data from a limited number of sensors. Mechanical Systems and Signal Processing 29, 310-327.

[37] Mariani, S., Ghisi, A., 2007. Unscented kalman filtering for nonlinear structural dynamics. Nonlinear Dynamics 49, $131-150$.

[38] Motro, R., Najari, S., Jouanna, P., 1987. Static and dynamic analysis of tensegrity systems, in: Shell and Spatial Structures: Computational Aspects. Springer, pp. 270-279.

[39] Obara, P., Kłosowska, J., Gilewski, W., 2019. Truth and myths about 2d tensegrity trusses. Applied sciences 9, 179.

[40] Oppenheim, I., Williams, W., 2000. Geometric effects in an elastic tensegrity structure. Journal of elasticity and the physical science of solids 59, 51-65.

[41] Oppenheim, I.J., Williams, W.O., 2001a. Vibration and damping in three-bar tensegrity structure. Journal of Aerospace Engineering 14, 85-91.

[42] Oppenheim, I.J., Williams, W.O., 2001b. Vibration of an elastic tensegrity structure. European Journal of MechanicsA/Solids 20, 1023-1031.

[43] Pan, P., Wang, T., Nakashima, M., 2016. Development of online hybrid testing: theory and applications to structural engineering. Elsevier / Butterworth Heinemann.

[44] Paul, C., Valero-Cuevas, F.J., Lipson, H., 2006. Design and control of tensegrity robots for locomotion. IEEE Transactions on Robotics 22, 944-957.

[45] Petrini, L., Maggi, C., Priestley, M.N., Calvi, G.M., 2008. Experimental verification of viscous damping modeling for inelastic time history analyzes. Journal of Earthquake Engineering 12, 125-145.

[46] Puthanpurayil, A.M., Dhakal, R.P., Carr, A.J., 2011. Modelling of in-structure damping: A review of the state-of-the-art, in: Proc. Ninth Pacific Conf. Earthquake Engineering, [Online]. Paper.

[47] Quirant, J., Kazi-Aoual, M., Motro, R., 2003. Designing tensegrity systems: the case of a double layer grid. Engineering Structures 25, 1121-1130. doi 10.1016/s0141-0296(03)00021-x 
48] Rhode-Barbarigos, L., Ali, N.B.H., Motro, R., Smith, I.F., 2010. Designing tensegrity modules for pedestrian bridges. Engineering Structures 32, 1158-1167. doi:10.1016/j.engstruct.2009.12.042

[49] Sabouni-Zawadzka, A., Gilewski, W., et al., 2018. Inherent properties of smart tensegrity structures. Applied Sciences 8, 787.

[50] Santos, F.L.M.D., Peeters, B., Lau, J., Desmet, W., Goes, L.C.S., 2015. The use of strain gauges in vibration-based damage detection. Journal of Physics: Conference Series 628, 012119. doi 10.1088/1742-6596/628/1/012119

[51] Sen, S., Bhattacharya, B., 2016. Progressive damage identification using dual extended kalman filter. Acta Mechanica 227, 2099-2109. doi 10.1007/s00707-016-1590-9

[52] Sen, S., Crinière, A., Mevel, L., Cérou, F., Dumoulin, J., 2018. Seismic-induced damage detection through parallel force and parameter estimation using an improved interacting particle-kalman filter. Mechanical Systems and Signal Processing $110,231-247$

[53] Skelton, R.E., Adhikari, R., Pinaud, J.., Waileung Chan, Helton, J.W., 2001. An introduction to the mechanics of tensegrity structures, in: Proceedings of the 40th IEEE Conference on Decision and Control (Cat. No.01CH37228), pp. 4254-4259 vol.5.

[54] Snelson, K., 1973. Tensegrity Masts.

[55] Subbaraj, K., Dokainish, M., 1989. A survey of direct time-integration methods in computational structural dynamics-ii. implicit methods. Computers \& Structures 32, 1387-1401.

56] Sultan, C., 2009a. Designing structures for dynamical properties via natural frequencies separation: Application to tensegrity structures design. Mechanical systems and signal processing 23, 1112-1122.

[57] Sultan, C., 2009b. Tensegrity: 60 years of art, science, and engineering. Advances in applied mechanics 43, $69-145$.

[58] Sultan, C., 2010. Proportional damping approximation using the energy gain and simultaneous perturbation stochastic approximation. Mechanical Systems and Signal Processing 24, 2210-2224.

[59] Sultan, C., 2013a. Decoupling approximation design using the peak to peak gain. Mechanical Systems and Signal Processing 36, 582-603.

60] Sultan, C., 2013b. Stiffness formulations and necessary and sufficient conditions for exponential stability of prestressable structures. International Journal of Solids and Structures 50, 2180-2195.

[61] Sultan, C., Corless, M., Skelton, R.E., 2000. Tensegrity flight simulator. Journal of Guidance, Control, and Dynamics 23, 1055-1064.

[62] Sultan, C., Corless, M., Skelton, R.T., 1999. Peak-to-peak control of an adaptive tensegrity space telescope, in: Smart Structures and Materials 1999: Mathematics and Control in Smart Structures, International Society for Optics and Photonics. pp. 190-201.

[63] Sultan, C., Skelton, R., 1997. Integrated design of controllable tensegrity structures. Adaptive structures and material systems-1997, 27-35.

[64] Sultan, C., Skelton, R., 2003. Deployment of tensegrity structures. International Journal of Solids and Structures 40, 4637-4657. doi 10.1016/s0020-7683(03)00267-1

[65] Sultan, C., Skelton, R.T., 1998. Tendon control deployment of tensegrity structures, in: Smart Structures and Materials 1998: Mathematics and Control in Smart Structures, International Society for Optics and Photonics. pp. 455-466.

[66] Sychterz, A.C., Smith, I.F., 2018. Using dynamic measurements to detect and locate ruptured cables on a tensegrity structure. Engineering Structures 173, 631-642.

[67] Tibert, A., Pellegrino, S., 2003. Review of form-finding methods for tensegrity structures. International Journal of Space Structures 18, 209-223.

[68] Tibert, G., 2002. Deployable tensegrity structures for space applications. Ph.D. thesis. KTH.

[69] Tran, H.C., Lee, J., 2011. Form-finding of tensegrity structures with multiple states of self-stress. Acta mechanica 222, 131.

[70] Yang, S., Sultan, C., 2016. Modeling of tensegrity-membrane systems. International Journal of Solids and Structures 82, $125-143$.

[71] Zghal, M., Mevel, L., Del Moral, P., 2014. Modal parameter estimation using interacting kalman filter. Mechanical Systems and Signal Processing 47, 139-150.

[72] Zhang, J., Ohsaki, M., 2007. Stability conditions for tensegrity structures. International journal of solids and structures $44,3875-3886$.

\section{Appendix A. Form-finding of statically stable tensegrity}

To find the initial statically stable configuration of tensegrity, a Force Density Method based algorithm is utilized that optimizes force density coefficients, $\mathbf{p}$, of the member elements to obtain initial coordinates, $\mathbf{X}_{\text {est }}$, of the tensegrity. Along with the optimization of force density coefficients, global stability criteria [72] (cf. lines 12, 15, Algorithm 2) are also introduced to obtain a stable tensegrity configuration. While constructing a physical tensegrity it has been noticed that the bars tend to buckle under self-stress. To avoid such a situation, local stability criteria (cf. lines 18, 19. Algorithm 2) of buckling failure $\left(\mathbf{p}_{\text {bars }}<\mathbf{p}_{\text {critical }}\right.$ ) 


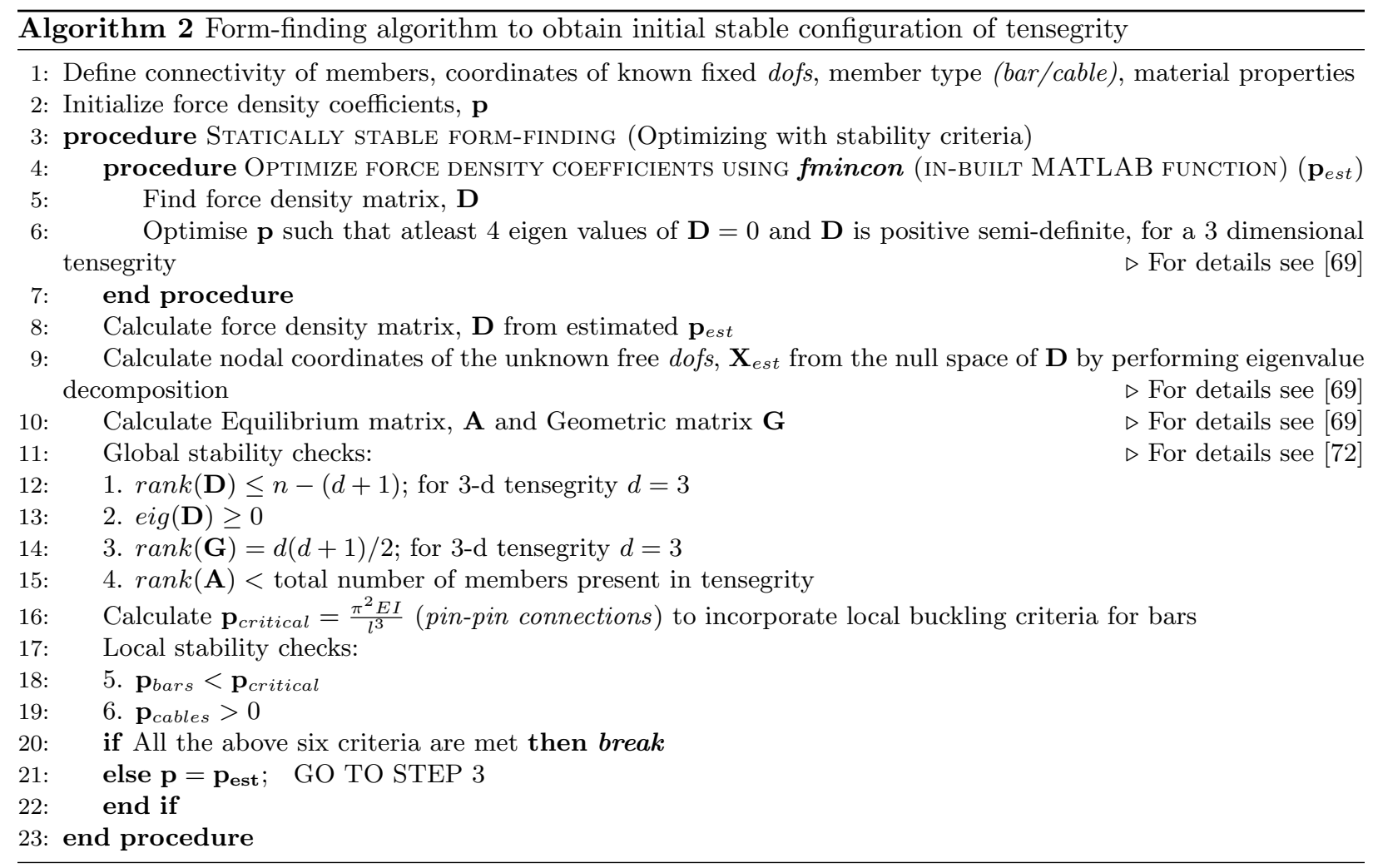

\section{Appendix B. Explicit Newmark-beta method: incremental formulation}

Algorithm 3 presents the pseudo-code for explicit Newmark-beta method [15] utilized in this study.

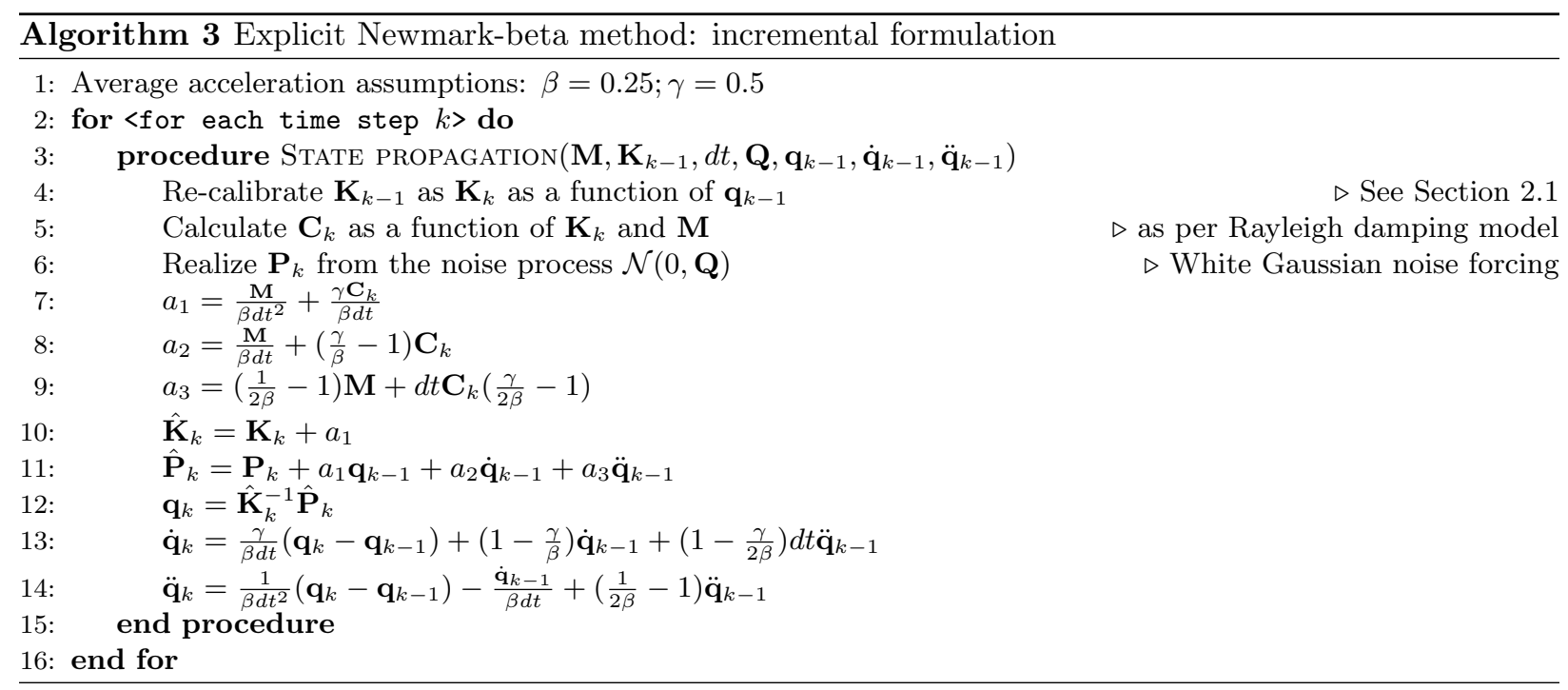

